\title{
ZioLib: a Parallel I/O Library
}

\author{
WoO-Sun YAng And Chris Ding \\ Computational Research Division \\ Lawrence Berkeley National Laboratory \\ University of California, Berkeley, CA 94720
}

\section{Contents}

1 Introduction 5

2 ZioLib Features 5

3 Source Files 6

4 How to Use: Quick Guide $\quad 7$

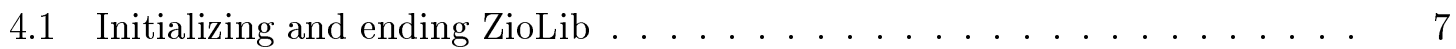

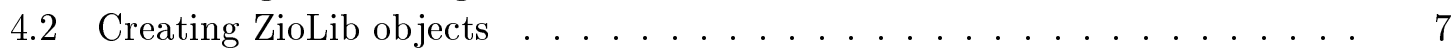

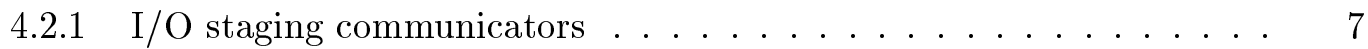

4.2 .2 Distributed array descriptors . . . . . . . . . . . . 7

4.2.3 List of distributed array descriptors . . . . . . . . . . . . . 8

4.2 .4 Others .............................. 9

4.3 Unformatted direct- or sequential-access $\mathrm{I} / \mathrm{O} \ldots \ldots \ldots \ldots$

4.4 NetCDF I/O . . . . . . . . . . . . . . . . . . . . . . . . . 10

5 Routine/Function Prologues $\quad 12$

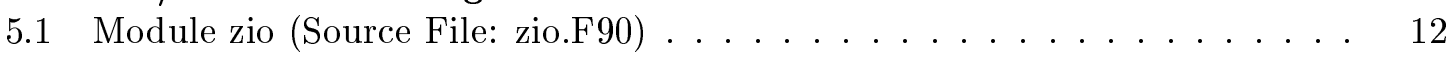

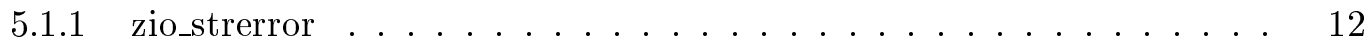

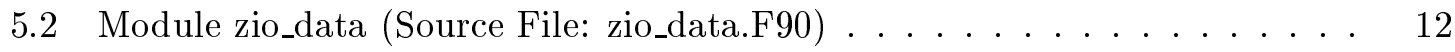

5.2 .1 zio_init . . . . . . . . . . . . . . . . . . 15

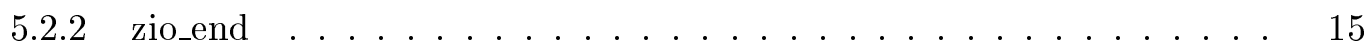

5.2 .3 zio_add_to_list . . . . . . . . . . . . . . . . . 16

5.2 .4 zio_check_decomp . . . . . . . . . . . . . . . 16

5.2 .5 zio_check_distarray . . . . . . . . . . . . . . . . . . . . . . 16

5.2 .6 zio_check_filedesc . . . . . . . . . . . . . . . . . . 17

5.2 .7 zio_check_iocomm . . . . . . . . . . . . . . . . . . . 17

5.2 .8 zio_data_strerror . . . . . . . . . . . . . . . . . . . . . . . 17

5.2 .9 zio_destroy_decomp . . . . . . . . . . . . . . . . . . 18

5.2 .10 zio_destroy_distarray . . . . . . . . . . . . . . . . . . . . . . . . 18

5.2 .11 zio_destroy_iocomm . . . . . . . . . . . . . . . . . . . 18

5.2 .12 zio_destroy_listentry . . . . . . . . . . . . . . . . . . . . . . 19

5.2 .13 zio_find_locnx . . . . . . . . . . . . . . . . . . . . . . . . 19

5.2 .14 zio_get_comm . . . . . . . . . . . . . . . . . . . . 19

5.2 .15 zio_get_coords . . . . . . . . . . . . . . . . 20 
5.2 .16 zio_get_distarray . . . . . . . . . . . . . . . . . . . 20

5.2 .17 zio_get_errtype . . . . . . . . . . . . . . 20

5.2 .18 zio_get_fileid . . . . . . . . . . . . ........ 21

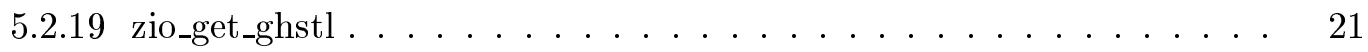

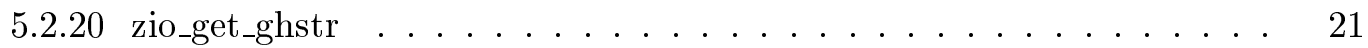

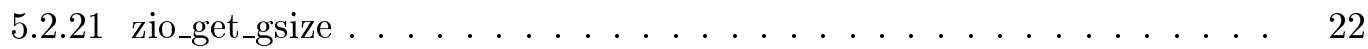

5.2 .22 zio_get_index_order . . . . . . . . . . . . . . . . . 22

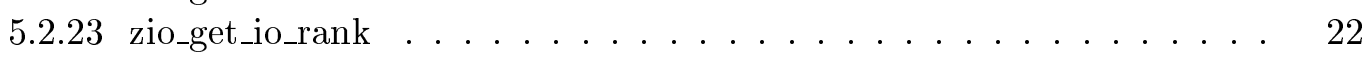

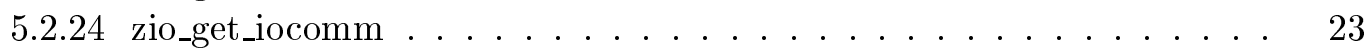

5.2 .25 zio_get_is_iope . . . . . . . . . . . . . . . . . 23

5.2 .26 zio_get_loff . . . . . . . . . . . . . . . . . . . 23

5.2 .27 zio_get_loff_me . . . . . . . . . . . . . . . . . . . 24

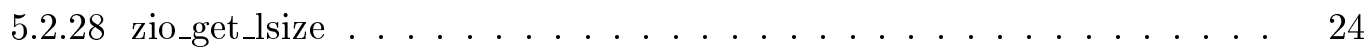

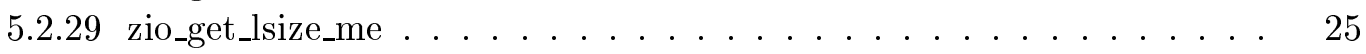

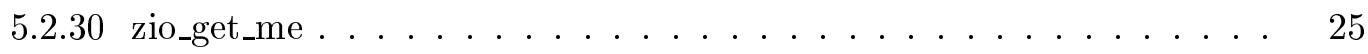

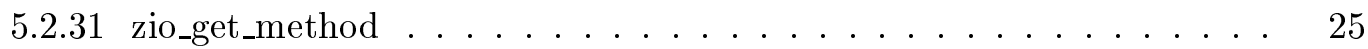

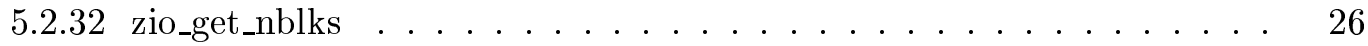

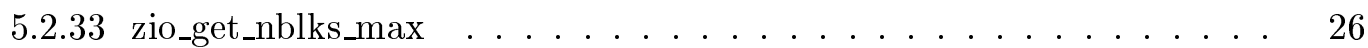

5.2 .34 zio_get_ndims_decomp . . . . . . . . . . . . . . 27

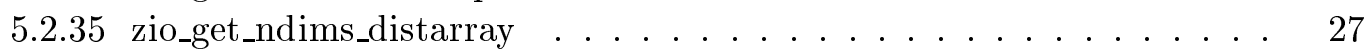

5.2 .36 zio_get_ndims_par . . . . . . . . . . . . . . . . . . . . . 27

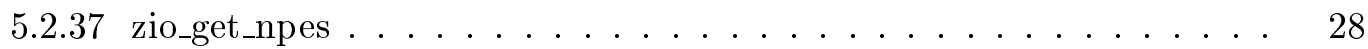

5.2 .38 zio_get_niopes . . . . . . . . . . . . . . . . . . . . . . 28

5.2 .39 zio_get_ranks_cmp ........................... 28

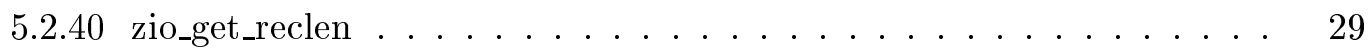

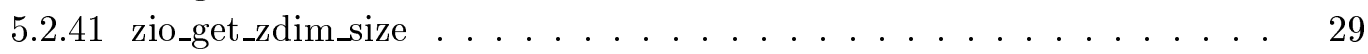

5.2 .42 zio_get_zio_comm . . . . . . . . . . . . . . . . . . . . . . . . 29

5.2 .43 zio_get_zio_initialized . . . . . . . . . . . . . . . 30

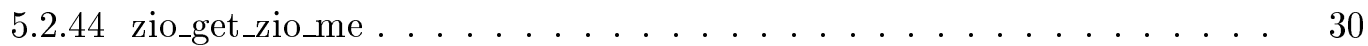

5.2 .45 zio_get_zio_npes . . . . . . . . . . . . . . . . . . 30

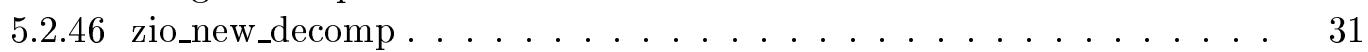

5.2 .47 zio_new_distarray . . . . . . . . . . . . . . . . . . . . . . . . . . . . . . 31

5.2 .48 zio_define_distarray . . . . . . . . . . . . . . . . . 32

5.2 .49 zio_import_distarray . . . . . . . . . . . . . . . . . 32

5.2 .50 zio_new iocomm . . . . . . . . . . . . . . . . . . . . . 33

5.2 .51 zio_new_iocomm_incl . . . . . . . . . . . . . . . . . 33

5.2 .52 zio_new_iocomm_member . . . . . . . . . . . . . 34

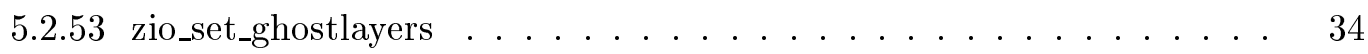

5.2 .54 zio_set_index_order . . . . . . . . . . . . . . . . . . . . . . . . . 34

5.2 .55 zio_set_ndims_par . . . . . . . . . . . . . . . 35

5.3 Module zio_remap (Source File: zio_remap.F90) . . . . . . . . . . . . . 35

5.3 .1 zio_from_iopes_double . . . . . . . . . . . . . 36

5.3 .2 zio_from_iopes_int . . . . . . . . . . . . . . . . . 36

5.3 .3 zio_from_iopes_real . . . . . . . . . . . . . . . . . . . . . . . . 37

5.3 .4 zio_to_iopes_double . . . . . . . . . . . . . . . . . . . . 37

5.3 .5 zio_to_iopes_int . . . . . . . . . . . . . . . . . . 38

5.3 .6 zio_to_iopes_real . . . . . . . . . . . . . . . . . . . 38

5.4 Module zio_binary (Source File: zio_binary.F90) . . . . . . . . . . . . 39 
5.4 .1 zio_uf_close . . . . . . . . . . . . . . . . . . . . . . 39

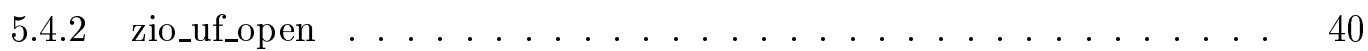

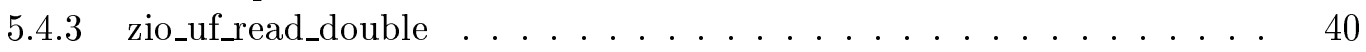

5.4 .4 zio_uf_read_double_name . . . . . . . . . . . . . . . . . . 41

5.4 .5 zio_uf_read_int . . . . . . . . . . . . . . . . . . 41

5.4 .6 zio_uf_read_int_name . . . . . . . . . . . . . . . . . . . 42

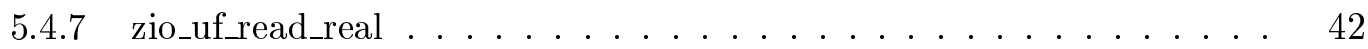

5.4 .8 zio_uf_read_real_name . . . . . . . . . . . . . . . . 43

5.4 .9 zio_uf_strerror . . . . . . . . . . . . . . . . . . . . 43

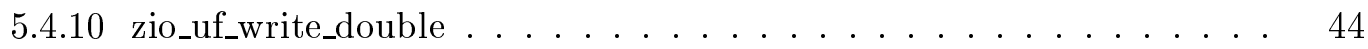

5.4 .11 zio_uf_write_double_name . . . . . . . . . . . . . . . 44

5.4 .12 zio_uf_write_int . . . . . . . . . . . . . . . . 45

5.4 .13 zio_uf_write_int_name . . . . . . . . . . . . . . . 45

5.4 .14 zio_uf_write_real . . . . . . . . . . . . . . . . 46

5.4 .15 zio_uf_write_real_name . . . . . . . . . . . . . . . 46

5.5 Module zio_netcdf77 (Source File: zio_netcdf77.F90) . . . . . . . . . . . 47

5.5 .1 zio_nf_comm . . . . . . . . . . . . . . . . . 47

5.5 .2 zio_nf__create . . . . . . . . . . . . . . . 47

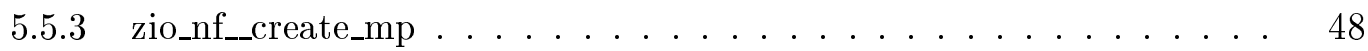

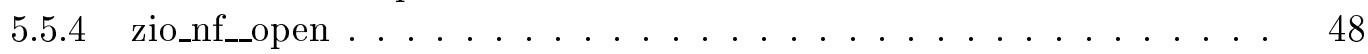

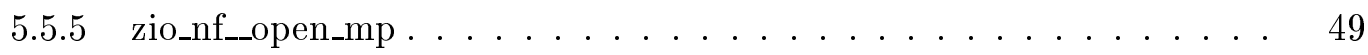

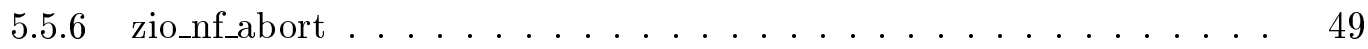

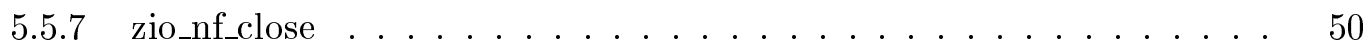

5.5 .8 zio_nf_copy_att . . . . . . . . . . . . . . . 50

5.5 .9 zio_nf_create . . . . . . . . . . . . . . . 50

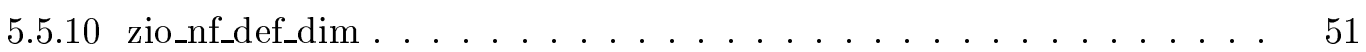

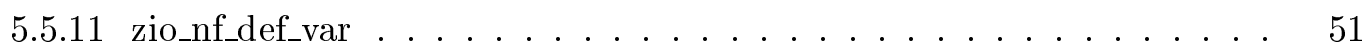

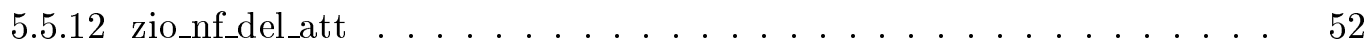

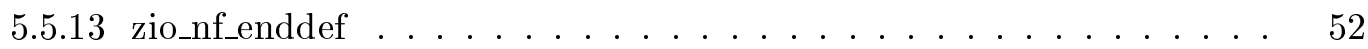

5.5 .14 zio_nf_get_att_double . . . . . . . . . . . . . . 52

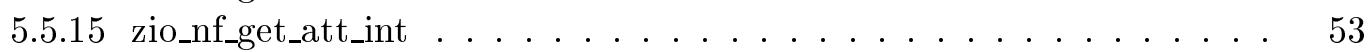

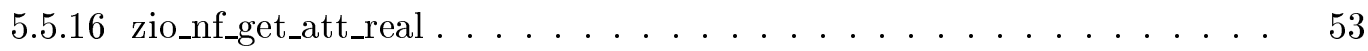

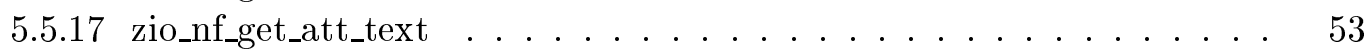

5.5 .18 zio_nf_get_var_double . . . . . . . . . . . . . . . 54

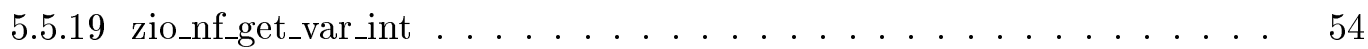

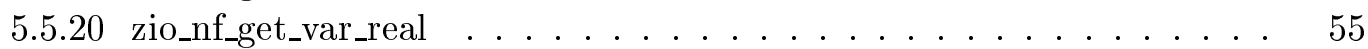

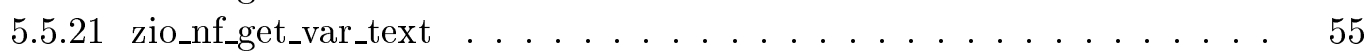

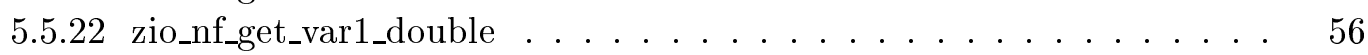

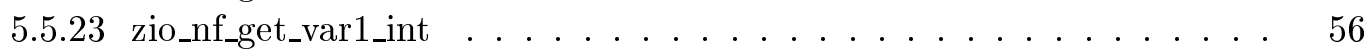

5.5 .24 zio_nf_get_var1_real . . . . . . . . . . . . . . . . 56

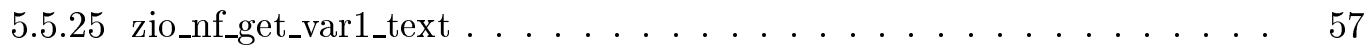

5.5 .26 zio_nf_get_vara_double . . . . . . . . . . . . . . 57

5.5 .27 zio_nf_get_vara_int . . . . . . . . . . . . . . . . 58

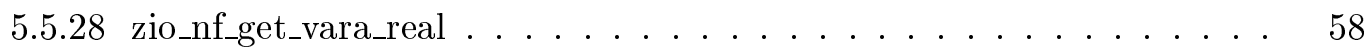

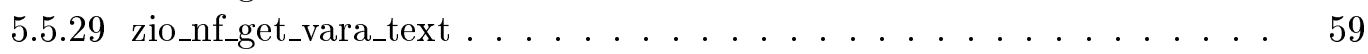

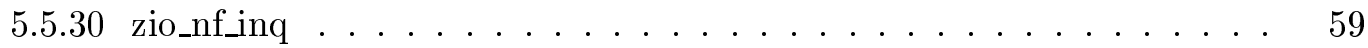

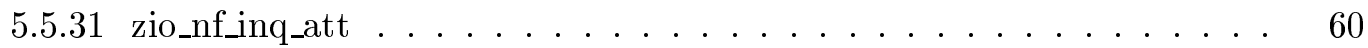

5.5 .32 zio_nf_inq_attid . . . . . . . . . . . . . . 60 
5.5 .33 zio_nf_inq_attlen . . . . . . . . . . . . . . . . . . 61

5.5 .34 zio_nf_inq_attname .......................... 61

5.5 .35 zio_nf_inq_atttype . . . . . . . . . . . . . . . . 62

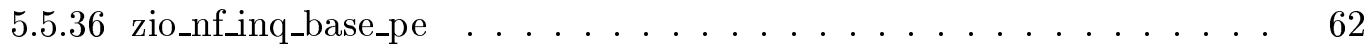

5.5.37 zio_nf_inq_dim . . . . . . . . . . . . . . . . . . 62

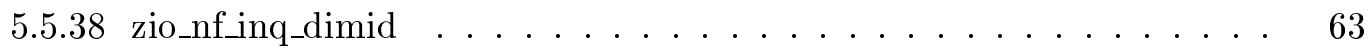

5.5 .39 zio_nf_inq_dimlen . . . . . . . . . . . . . . . . . 63

5.5 .40 zio_nf_inq_dimname . . . . . . . . . . . . . . . . 64

5.5 .41 zio_nf_inq_libvers . . . . . . . . . . . . . . . . . . . 64

5.5 .42 zio_nf_inq_natts . . . . . . . . . . . . . . . . . . . . 64

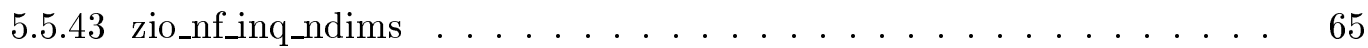

5.5 .44 zio_nf_inq_nvars . . . . . . . . . . . . . . . . . 65

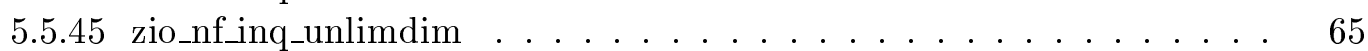

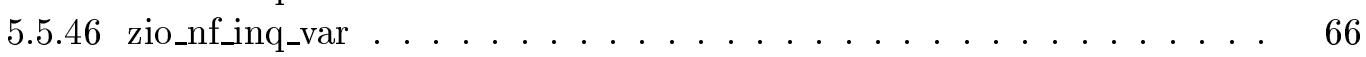

5.5 .47 zio_nf_inq_vardimid . . . . . . . . . . . . . . . . 66

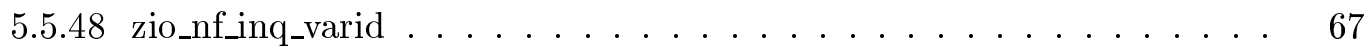

5.5 .49 zio_nf_inq_varname . . . . . . . . . . . . . . . . 67

5.5.50 zio_nf_inq_varnatts . . . . . . . . . . . . . . . 67

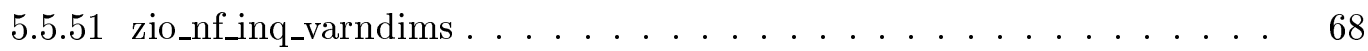

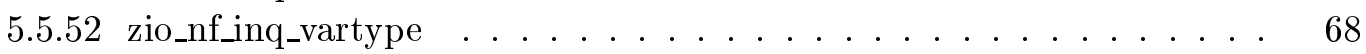

5.5 .53 zio_nf_open . . . . . . . . . . . . . . . . . . . . 69

5.5 .54 zio_nf_put_att_double . . . . . . . . . . . . . . . 69

5.5 .55 zio_nf_put_att_int . . . . . . . . . . . . . . . . . 69

5.5.56 zio_nf_put_att_real . . . . . . . . . . . . . . . 70

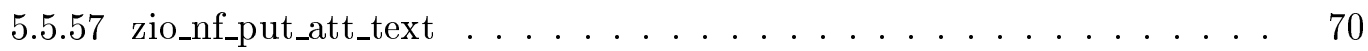

5.5 .58 zio_nf_put_var_double . . . . . . . . . . . . . . 71

5.5 .59 zio_nf_put_var_int . . . . . . . . . . . . . . . 71

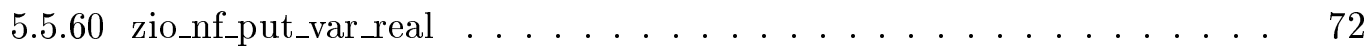

5.5 .61 zio_nf_put_var_text . . . . . . . . . . . . . . . . 72

5.5.62 zio_nf_put_var1_double . . . . . . . . . . . . . . . 72

5.5 .63 zio_nf_put_var1_int . . . . . . . . . . . . . . 73

5.5 .64 zio_nf_put_var1_real . . . . . . . . . . . . . . . . . . . . . 73

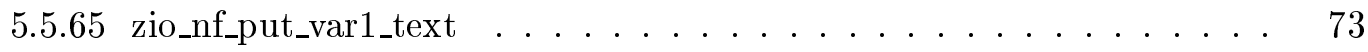

5.5 .66 zio_nf_put_vara_double . . . . . . . . . . . . . . . . . 74

5.5 .67 zio_nf_put_vara_int . . . . . . . . . . . . . . . . . 74

5.5.68 zio_nf_put_vara_real . . . . . . . . . . . . . . . . . . 75

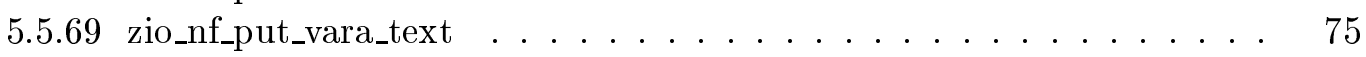

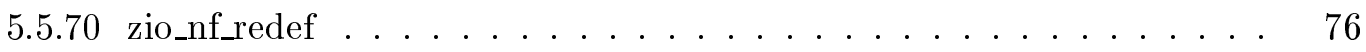

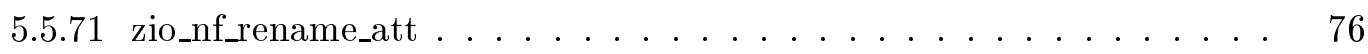

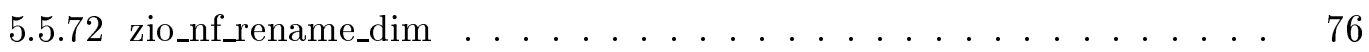

5.5 .73 zio_nf_rename_var . . . . . . . . . . . . . . . . . 77

5.5 .74 zio_nf_set_base_pe . . . . . . . . . . . . . . . . . . . . . . . . 77

5.5 .75 zio_nf_set_fill . . . . . . . . . . . . . . . . . . . . . . . . 77

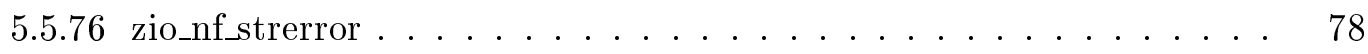

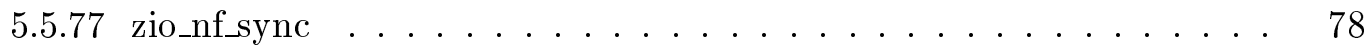




\section{Introduction}

In a distributed memory parallel environment, many applications rely on a serial I/O strategy, where the global array is gathered on a single MPI process and then written out to a file. I/O performance with this approach is largely limited by single process' I/O bandwidth. Even when parallel I/O is used, satisfactory parallel scaling is not always observed. It is because in many applications fields are not necessarily in a most favorable parallel decomposition for I/O. The best I/O rates are obtained when a field is decomposed with respect to the array's last dimension (referred to here as "Z").

Another situation often encountered in many applications is that a field in CPU resident memory is in one index order but must be stored in a disk file in another order. Changing index orders can complicate a parallel I/O implementation and slow down I/O.

ZioLib facilitates an efficient parallel I/O for arrays in such situations. In case of a write, ZioLib remaps a distributed field into a Z-decomposition on a subset of processes (which will be called the $I / O$ staging processes) and from there writes to a disk file in parallel (see Figure 1). In this Z-decomposition, the data layout of the remapped array on the staging processes' memory is the same as on disk, thus only block data transfer occurs during parallel I/O, achieving maximum efficiency. In case of a read the steps are reversed to build the required distributed arrays on the computational processes.

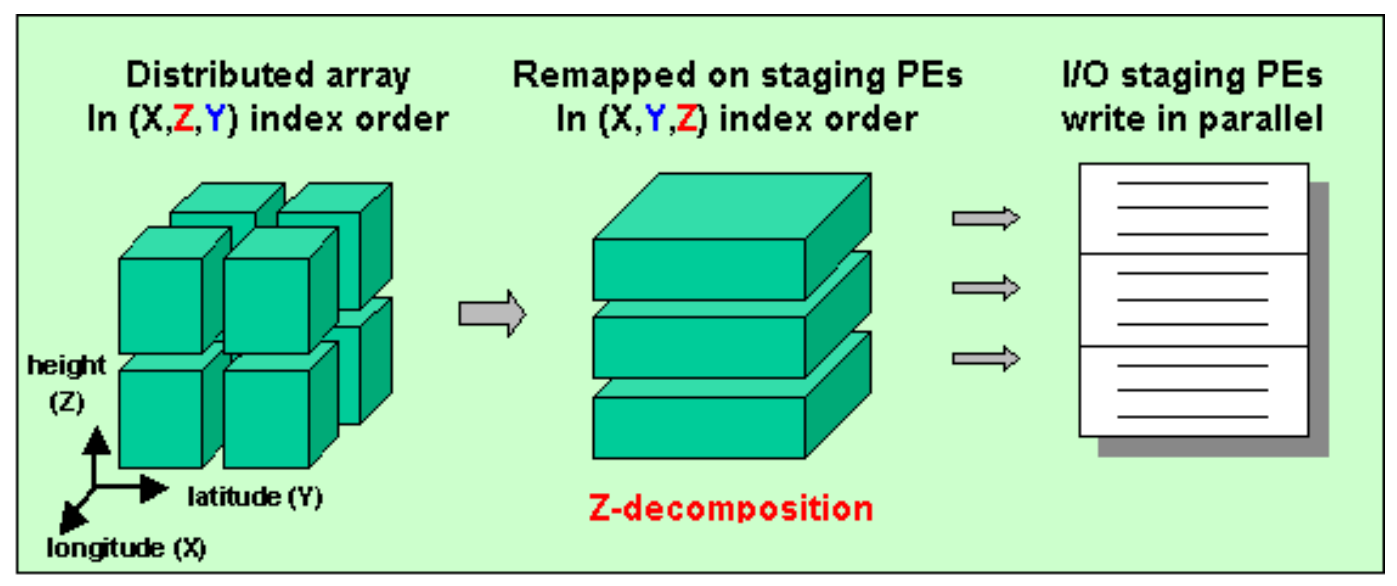

Figure 1: Writing the global field of distributed array $\mathrm{a}(\mathrm{X}, \mathrm{Z}, \mathrm{Y})$ to a disk file in $(\mathrm{X}, \mathrm{Y}, \mathrm{Z})$ index order using three I/O staging processes.

Please see more information at http://www.nersc.gov/research/SCG/acpi/ZioLib/.

\section{ZioLib Features}

- A set of Fortran 90 modules supporting

- netCDF I/O (wrappers for serial and parallel library routines)

- direct-access unformatted I/O (serial and parallel)

- sequential-access unformatted I/O (serial)

- Read or write arrays of an arbitrary number of dimensions 
- Support integer $* 4$, real $* 4$ and real $* 8$ data types

- Read or write in any array index order

- Work with any block parallel decompositions

- A process' subdomain can be made of multiple disjoint rectangular blocks

- Can handle ghost nodes

- Use only MPI-1 routines for greater portability

ZioLib works for regular rectangular block-decompositions. It also works for more general data decomposition cases where each process' local subdomain consists of a number of separate rectangular blocks. Such a more complex decomposition is not uncommon in many application codes. See the test codes bintest4.F90 and nftest4.F90 included in the source code tar file to see how to use ZioLib functions in such a situation.

The parallel netCDF library for which ZioLib provides wrappers is the one that is being developed at LBNL/NERSC (http://hpcf.nersc.gov/software/libs/io/netcdf/sp/pusage.html). Since staging processes can be set flexibly and the required remapping of data is all performed by ZioLib, we notice a few distinct advantages with ZioLib:

- Writes/reads are done in large blocks (no seeks) in parallel

- Robust I/O performance is achieved regardless of parallel decomposition types

- Compared to serial I/O, memory limitations on a process can be relieved because global field is now constructed on multiple staging processes instead of single root process

- Congestion on I/O server nodes can be relieved (because the number of disk-accessing staging processes can be controlled)

- Gather/scatter/transpose is eliminated from user codes

\section{Source Files}

Source files, zio.h, zio_data.F90, zio_remap.F90, zio_binary.F90, zio_netcdf77.F90, and zio.F90, are available at http://www.nersc.gov/research/SCG/acpi/ZioLib/. These codes can be compiled in either fixed or free source form.

The tar file, ziolib.tar, also contains a number of test codes: bintest1.F90, ..,bintest4 .F90 for Fortran unformatted I/O; nftest1.F90,...,nftest4.F90 for netCDF I/O. A short description about the tests can be found in the Readme file contained in the tar file. The test codes are in free source form.

As of now, ZioLib has been successfully tested on the following platforms:

- IBM SP

- SGI Origin

- Linux cluster with pgf90 (Portland Group) and lf95 (Lahey) Fortran compilers

To use the parallel netCDF library for ZioLib on an IBM SP (if it is installed on the machine), define ZIO_NETCDF_PAR_AIX (i.e., '\#define ZIO_NETCDF_PAR_AIX') in zio.h. Otherwise, the serial netCDF library will be used. 


\section{How to Use: Quick Guide}

Users can access ZioLib procedures by including 'use zio' in their codes.

Most of the ZioLib procedures return an integer error code. When no error is found during invocation of a procedure, zio_noerr (0) is returned. When an error is encounted, an error message can be obtained by calling zio_strerror(errcode[,errtype]). It returns a character string of length 80 .

\subsection{Initializing and ending ZioLib}

To be able to use ZioLib, MPI must be initialized first. Then ZioLib must be initialized with a call to zio_init with the MPI communicator used for computation as the argument. For example,

ret $=$ zio_init (mpi_comm_world)

To end ZioLib, zio_end() is called.

\subsection{Creating ZioLib objects}

\subsubsection{I/O staging communicators}

This is to designate a subset of the compute communicator processes as the ones that actually stage I/O. For serial I/O (e.g., sequential-access Fortran unformatted I/O) one can select the process 0 as the staging process. For parallel I/O, you can select a subset of processes from all the compute processes to achieve an optimal I/O bandwidth.

There are a few ways to create I/O staging communicators out of the compute communicator (the handle for the created I/O staging communicator object is returned in iocomm):

zio_new_iocomm (iocomm, $n$ ) staging communicator made of first $\mathrm{n}$ processes

zio_new_iocomm_incl (iocomm, $n$, ranks) staging communicator made of ranks $(1: n)$ processes

zio_new_iocomm_member(iocomm, is_iope) staging communicator made of the processes with is_iops $=$.true.

A single staging communicator object can be used for all subsequent I/O. But a flexible arrangement is possible. For example, if the maximum read and write rates can be obtained with different numbers of processes on a machine, you may want to define two I/O staging communicator objects, one for reads and the other for writes.

\subsubsection{Distributed array descriptors}

ZioLib needs to know how an array is distributed among processes. If distributed array's domain is made of nblks separate rectangular blocks, one way is to describe the distributed array is to specify each rectangular block's local grid size along each array dimension (1size(1), lsize(2), lsize(3), ..) and its grid offsets (offset(1), offset(2), offset $(3), \ldots)$ in the global array space: 
ret $=$ zio_new_distarray (distarray,nblks, ndims, offset, lsize)

It returns a distributed array descriptor in distarray.

In some case, not all processes have valid data assigned to them. For example, for 2-D surface data in a 3-D decomposition, some processes do not contain valid surface data. In such a case, make sure to set either nblks or lsize(:) to 0 if the current process does not contain valid data.

Another way to create a descriptor is to specify it via a parallel decomposition:

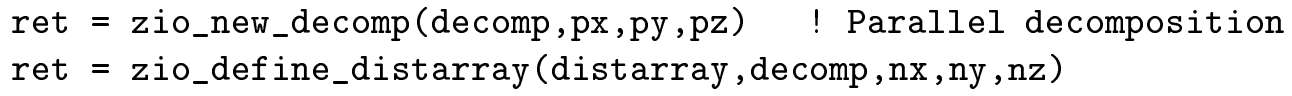

Here we are dealing with an array of global grid sizes nx, ny, and nz, block-distributed over $\mathrm{px}, \mathrm{py}$, and pz processes. Distributed arrays for up to 5-D parallel decompositions can be described in this way. Note, however, that there are some restrictions with this approach. First, it is assumed that process ranks change fastest along the $\mathrm{X}$-axis, then Y-axis, and then Z-axis (for MPI's Cartesian process topology, rank ordering is the opposite). When a grid size is not evenly divisible by the respective process count, it is assumed that the excess grid points (that is, the remainder) are evenly distributed among the lowest-rank processs. For example, if there are 26 grid points distributed among four processs, it is assumed that the local grid points for processes $0,1,2$, and 3 are $7,7,6$, and 6 , respectively.

If offsets, local sizes, ghost nodes, and index order are set in some routine (presumably provided by a code developer) and a user doesn't want to figure them out himself/herself, then the user can create a distributed array descriptor by simply providing the subroutine name:

ret $=$ zio_import_distarray (distarray,user_func)

where user func must be provided in the user code. See Section 5 for details.

With a distributed array discriptor ZioLib can figure out how to read or write a global array. ZioLib can also read or write non-distributed array replicated on all processes. To read or write such an array, the flag zio_replicated should be used as a descriptor.

\subsubsection{List of distributed array descriptors}

Distribued array descriptors can be conveniently stored in the descriptor list, and they can be retrieved at a later time with variable names (character strings):

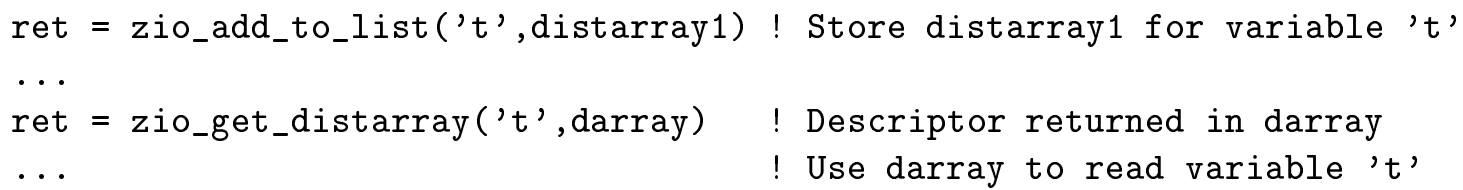

It is not necessary to use this feature of storing to and retrieving from the distributed array descriptor list, if a user remembers the descriptor for each field to be read or written. If this feature is used in conjuction with netCDF I/O, the variable name used for storing in the descriptor list must be the same as the netCDF variable name defined with zionnf_def_var. See Sections 4.3 and 4.4 . 


\subsubsection{Others}

Ghost layers for distributed arrays can be specified with zio_set_ghostlayers (distarray, ghstl, ghstr). It sets the numbers of ghost nodes of distributed array distarray. The ghost nodes preceding the real ones (LHS ghost nodes) must be provided as (ghst1(1), ghstl (2) , ...), and the ghost nodes after the real ones (RHS ghost nodes) as (ghstr(1), ghstr $(2), \ldots$ ) for all array dimensions. Files contain the real node values only.

If the output index order is different from the distributed array's local index order, one can set the output order with zio_set_index_order (distarray, order). If $\operatorname{order}(:)=(3,1,2)$, it means that the local array $t(i, j, k)$ will be stored in disk in $(j, k, i)$ order; or that the data in disk stored in $(j, k, i)$ order will be read into the local array as $t(i, j, k)$.

Actually ghost layers and output index order can be specified at the same time when a new distributed array descriptor is created with zio_new_distarray. They can be also reset with calls to the above functions after a descriptor was created.

For arrays of a small number of dimensions, you may not want to go with parallel I/O. You can control serial vs. parallel I/O for a certain file by specifying the minimum number of array dimensions for parallel I/O. This is set with a call to zio_set_ndims_par(filedesc, ndims_par). For example, if you issue zio_set_ndims_par(filedesc, 3), 1- and 2-D arrays will be written or read using a single staging process, no matter how many I/O staging processes are currently assigned.

\subsection{Unformatted direct- or sequential-access $\mathrm{I} / \mathrm{O}$}

A file is opened with

ret = zio_uf_open (filedesc, iocomm, unit,file,access, recl, ...)

Variables will be written to or read from the file file using unit number unit in access mode access, using the I/O staging communicator, iocomm. Note that unit, file, access, recl, etc. are the usual Fortran open statement arguments. Such an I/O channel is established and the handle for that is returned in filedesc.

When sequential-access is specified, the process 0 of the staging communicator will be the staging process for subsequent serial I/O.

Variables are written to or read from the file associated with the file descriptor filedesc using

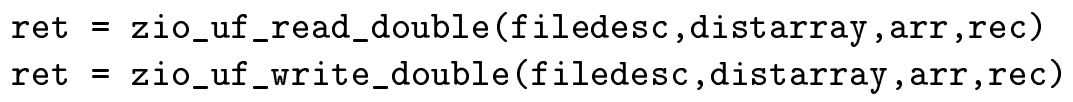

where $\mathrm{rec}$ is the global record number in the file (direct-access I/O; can be omitted for sequential-access I/O), distarray is the distributed array descriptor for the variable, and

arr is the buffer containing the local distributed data. If the current process's parallel subdomain is made of multiple separate blocks, arr contains all the local data contiguously one block after another, following the block order used to define distarray.

When only an array section of a variable is to be written or read, the section must be specified with istart (beginning indices; one-based) and icount (numbers of array elements) arrays. 
ret $=$ zio_uf_read_double(filedesc, distarray, arr, rec, istart, icount)
ret $=$ zio_uf_write_double(filedesc, distarray, arr, rec, istart, icount)

Note that istart and icount must be in the local array's index order (not the output index order, if those index orders are different).

If a distributed array descriptor is stored in the descriptor list, one can use instead of the descriptor the variable's name (that is, character string) that was used to store it in the list. See Section 4.2.3.

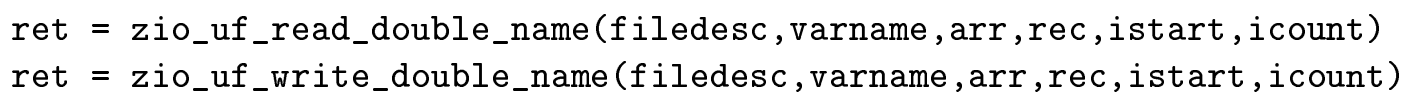

I/O for four-byte real and four-byte integer arrays can be similarly done with zio_uf_read_real, zio_uf_read_real_name, zio_uf_read_int, zio_uf_read_int_name, zio_uf_write_real, zio_uf_write_real_name, zio_uf_write_int, and zio_uf_read_int_name.

A file is closed with

ret $=$ zio_uf_close $($ filedesc $)$

\subsection{NetCDF I/O}

To create a netCDF file, one can use

ret $=$ zio_nf_create (filename, cmode, iocomm,filedesc)

which indicates that data will be written in the netCDF creation mode cmode (nf_clobber, nf_share, or nf_write) using the I/O staging communicator iocomm. This I/O channel is referred to as filedesc. Similarly a file can be open in the open mode omode with

ret $=$ zio_nf_open (filename, omode, iocomm,filedesc $)$

Many netCDF functions can be invoked in a similar way as the regular netCDF functions would be - only with a netCDF file ID replaced with a file descriptor:

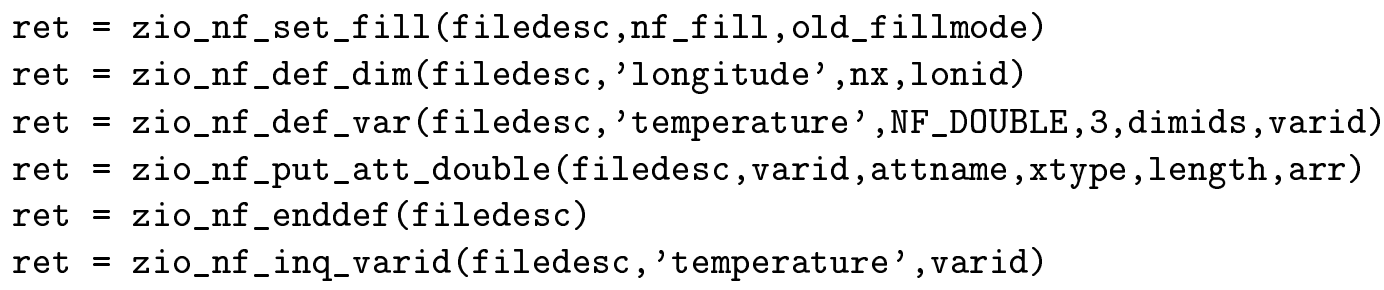

Variables are written or read using 


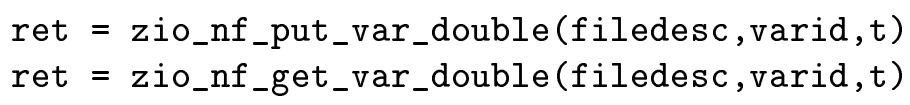

As in the case of the unformatted I/O, if the current process's parallel subdomain is made of multiple separate blocks, $t$ contains all the local data contiguously one block after another, following the block order used to define distarray.

Writing or reading an array section of a variable can be done with:

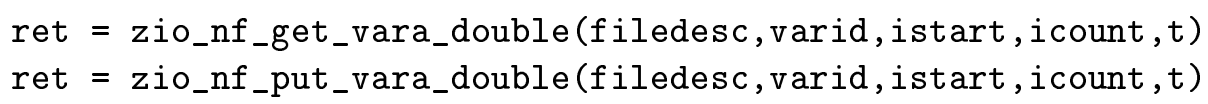

In the examples above, the distributed array descriptor for variable $t$ is assumed to be already added to the distributed array descriptor list using zio_add_to_list with the same variable name used for defining the netCDF variable (i.e., 'temperature'). (See Section 4.2.3.) If it is not on the list or a user does not want to use the feature of storing to and retrieving from the list, the argument distarray must be provided at the end of the argument list.

NOTE: In netCDF istart and icount used to refer only to the array section in the netCDF data space in disk, but not array's memory space. However, currently we let istart and icount refer to an array section in both the netCDF data and memory space. That is, an array section in netCDF data space is written from or read into the corresponding section of the global array.

istart and icount must be in the output index order. That is, they must be in the order specified when the netCDF variable is defined.

One thing to remember when using ZioLib netCDF functions is that a Fortran 90 module provides an explicit interface for module procedures. One consequence is that, if a dummy argument for a procedure is an array, the actual argument must be an array, too. (But array ranks of the actual and dummy arguments do not have to match if the dummy argument is an assumed-size array.) For example, if a single scalar value, say ' 0 ', is to be written with zio_nf_put_vara_double, the actual argument must be in an array form:

ret = zio_nf_put_vara_double (filedesc, varid, istart, icount, $(/ 0 /))$

This restriction must be observed in other netCDF routines, too. For example, one must write

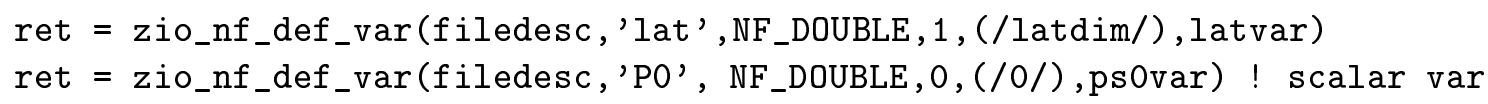

instead of zio_nf_def_var(filedesc, 'lat', NF_DOUBLE, 1, latdim, latvar) and zio_nf_def_var (filedesc , 'PO', NF DOUBLE, 0, 0, ps0var), respectively.

Currently the functions of the varm and vars families are not supported.

A file is closed with

ret $=$ zio_nf_close $($ filedesc $)$ 


\section{Routine/Function Prologues}

\subsection{Module zio (Source File: zio.F90)}

Module zio provides a single interface for all ZioLib procedures.

module zio

USES:

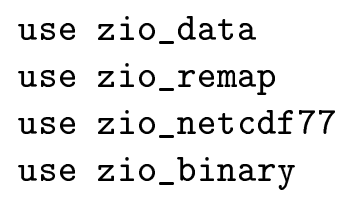

\subsection{1 zio_strerror}

INTERFACE:

function zio_strerror(errcode, errtype)

\section{DESCRIPTION:}

Returns an error message string of len=80 for error code errcode and error type errtype. If errtype is omitted, the error type set during execution of ZioLib procedures (zio_errtype) is used.

OUTPUT PARAMETERS:

character(len=80) : : zio_strerror

INPUT PARAMETERS:

integer, intent(in) : : errcode

integer, optional, intent(in) : : errtype

\subsection{Module zio_data (Source File: zio_data.F90)}

Module for ZioLib data and utility functions

INTERFACE:

module zio_data

DEFINED PARAMETERS: 


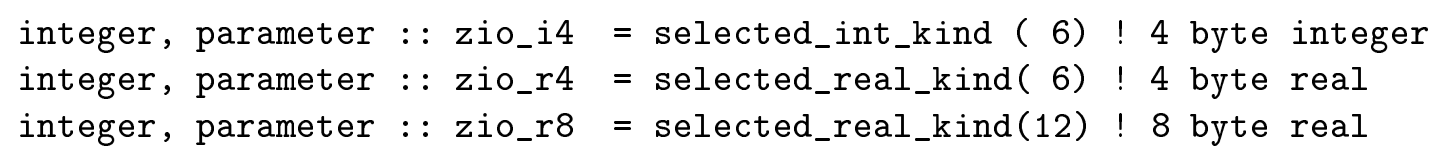

!... Maximum sizes

$\begin{array}{llll}\text { integer, parameter : zio_maxdims } & =100 & \text { ! Max dimensions } \\ \text { integer, parameter : zio_maxcomms }=100 & \text { ! Max I/0 staging comms } \\ \text { integer, parameter : : zio_maxdata } & =1000 & \text { ! Max distributed array objs } \\ \text { integer, parameter :: zio_maxblks }=100 & \text { ! Max rectangular blocks } \\ \text { integer, parameter : : zio_maxfiles }=100 & \text { ! Max file descriptors } \\ \text { integer, parameter : : zio_maxdecomp }=100 & \text { ! Max parallel decomps } \\ \text { integer, parameter :: zio_maxlist }=1000 & \text { ! Max list entries } \\ \text { integer, parameter : zio_maxchars }=128 & \text { ! Max character length }\end{array}$

!... I/O methods

integer, parameter : : zio_maxmethods = 3 ! Supported $\mathrm{I} / 0$ methods

integer, parameter : : zio_netcdf_io $=1$ ! For netCDF I/0

integer, parameter : : zio_direct_io = 2 ! For direct-access $\mathrm{I} / 0$

integer, parameter : : zio_sequential_io = 3 ! For sequential-access I/0

!... Flag for $\mathrm{I} / 0$ by root process only in case of replicated global array

integer, parameter : : zio_replicated $=-1$

!... Flag for undefined entity

integer, parameter : : zio_undefined = -99

!... Error codes

!... no error: zio_noerr $=$ nf_noerr $=$ mpi_success $=0$, iostat $=0$

integer, parameter :: zio_noerr $\quad=0$

integer, parameter : : zio_emax

$=-101$ ! Code range $\max$

integer, parameter : : zio_emin

$=-150$ ! Code range min

integer, parameter : : zio_error_allocate = zio_emin +0

integer, parameter : : zio_error_argument $\quad=$ zio_emin +1

integer, parameter : : zio_error_arraysection = zio_emin +2

integer, parameter : : zio_error_deallocate = zio_emin +3

integer, parameter : : zio_error_decomp = zio_emin +4

integer, parameter : : zio_error_distarray = zio_emin +5

integer, parameter : : zio_error_filedesc = zio_emin +6

integer, parameter : : zio_error_global_values = zio_emin +7

integer, parameter : : zio_error_imap $=$ zio_emin +8

integer, parameter : : zio_error_index_order $=$ zio_emin +9 


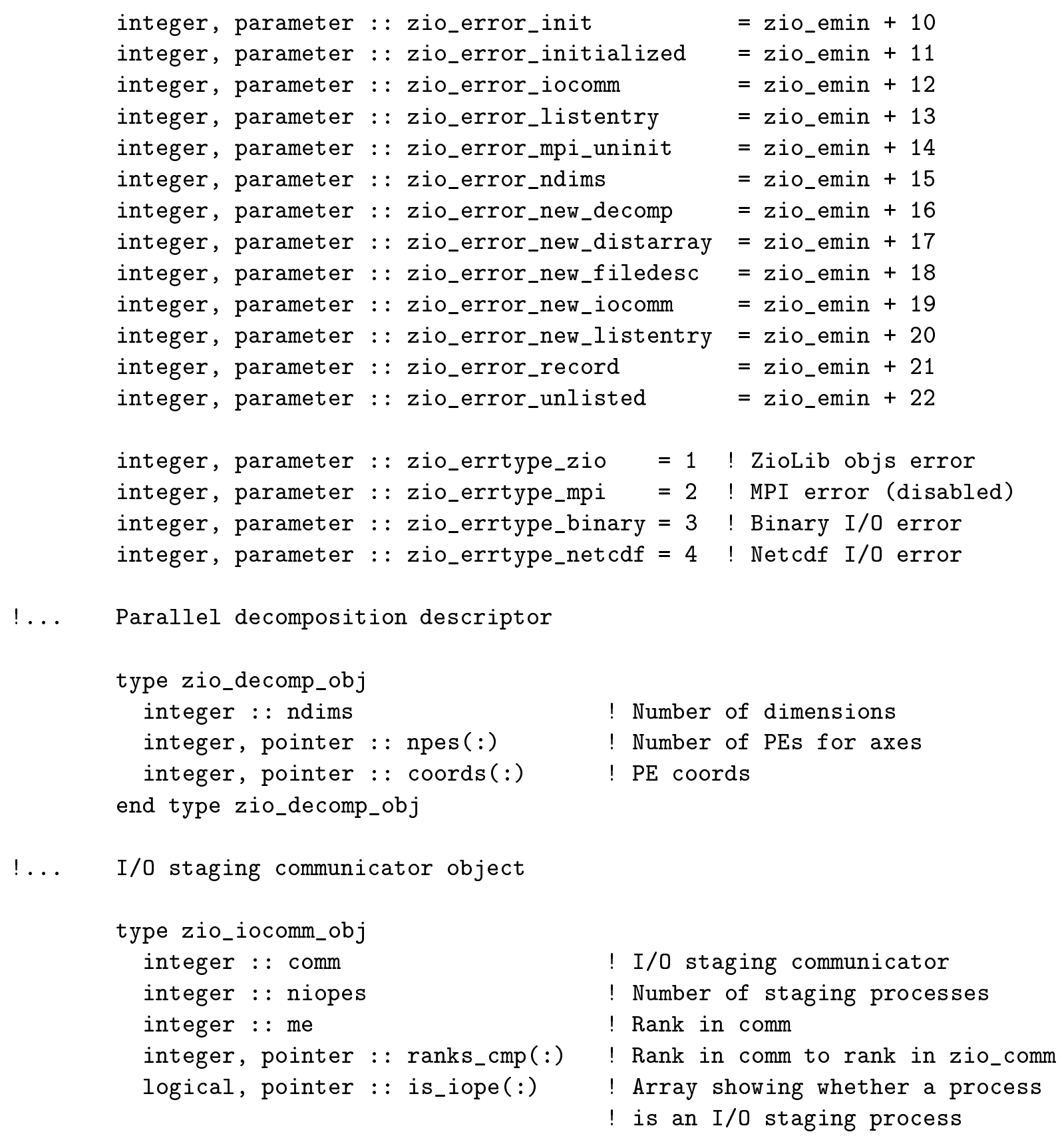




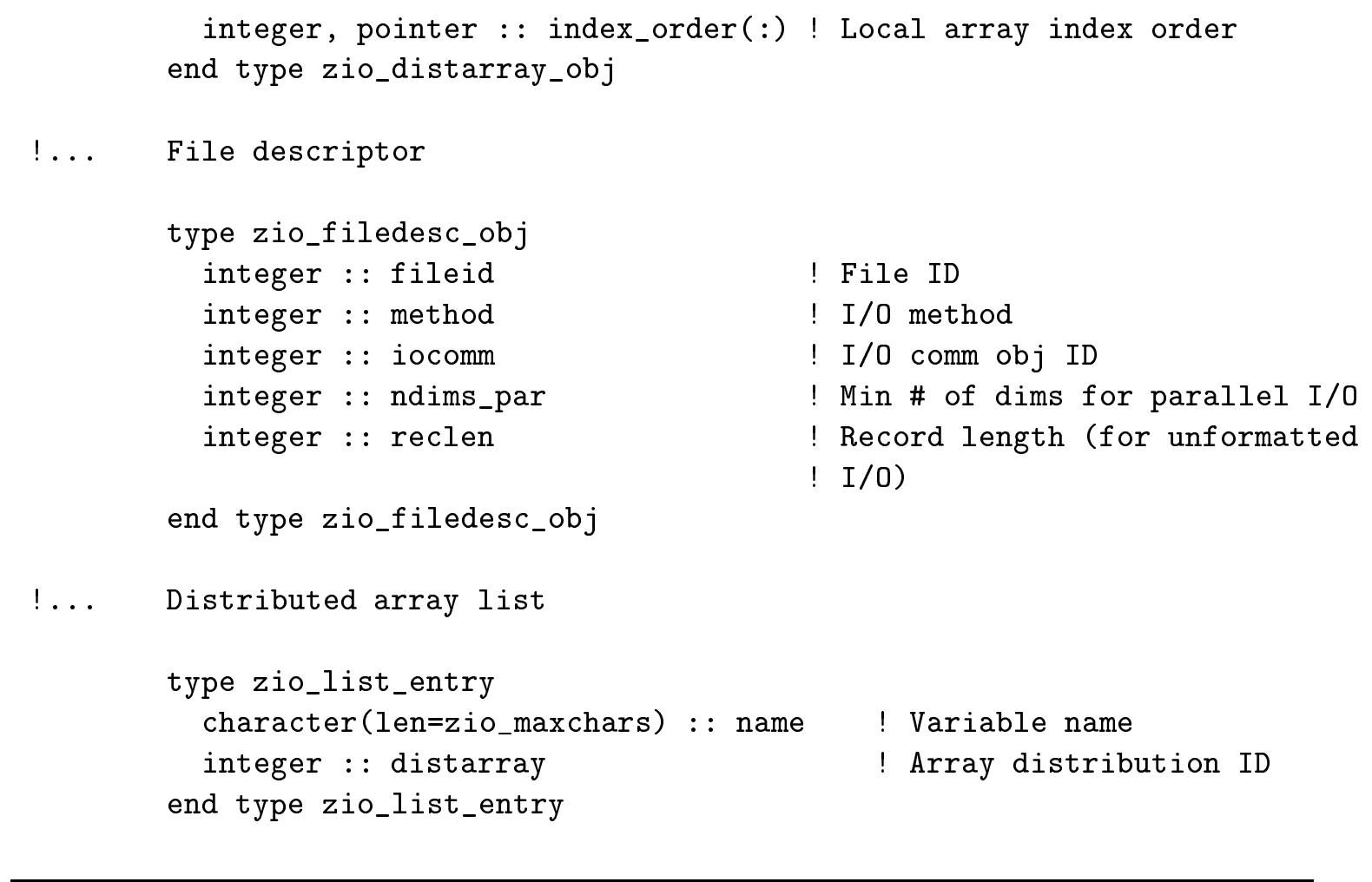

\subsection{1 zio_init}

INTERFACE:

integer function zio_init(comm)

DESCRIPTION:

Begin ZioLib.

INPUT PARAMETERS:
integer, intent(in) :: comm
! Compute communicator

\subsection{2 zio_end}

INTERFACE:

integer function zio_end()

DESCRIPTION:

End ZioLib 


\subsection{3 zio_add_to_list}

INTERFACE:

integer function zio_add_to_list(name,distarray)

DESCRIPTION:

Add a distributed array entry to the distributed array list

INPUT PARAMETERS:

character(len=*), intent(in) : : name

integer, intent(in) : : distarray

\subsection{4 zio_check_decomp}

INTERFACE:

integer function zio_check_decomp(decomp)

\section{DESCRIPTION:}

Checks whether decomp is a valid parallel decomposition descriptor.

INPUT PARAMETERS:

integer, intent(in) : : decomp

\subsection{5 zio_check_distarray}

INTERFACE:

integer function zio_check_distarray(distarray)

DESCRIPTION:

Checks whether distarray is a valid distributed array descriptor.

INPUT PARAMETERS:

integer, intent(in) : : distarray 


\subsection{6 zio_check_filedesc}

INTERFACE:

integer function zio_check_filedesc(filedesc)

\section{DESCRIPTION:}

Checks whether filedesc is a valid file descriptor.

INPUT PARAMETERS:

integer, intent(in) : : filedesc

\subsection{7 zio_check_iocomm}

INTERFACE:

integer function zio_check_iocomm(iocomm)

DESCRIPTION:

Checks whether iocomm is a valid I/O staging communicator object.

INPUT PARAMETERS:

integer, intent(in) :: iocomm

\subsection{8 zio_data_strerror}

INTERFACE:

function zio_data_strerror(errcode)

DESCRIPTION:

Returns an error message of length 80 for error code errcode that is in general related to managing ZioLib objects.

OUTPUT PARAMETERS:

character(len=80) : : zio_data_strerror

INPUT PARAMETERS:

integer, intent(in) :: errcode 


\subsection{9 zio_destroy_decomp}

INTERFACE:

integer function zio_destroy_decomp(decomp)

DESCRIPTION:

Destroy parallel decomposition descriptor decomp.

INPUT PARAMETERS:

integer, intent(in) : : decomp

\subsubsection{0 zio_destroy_distarray}

INTERFACE:

integer function zio_destroy_distarray(distarray)

DESCRIPTION:

Destroy distributed array descriptor distarray.

INPUT PARAMETERS:

integer, intent(in) : : distarray

\subsubsection{1 zio_destroy_iocomm}

INTERFACE:

integer function zio_destroy_iocomm(iocomm)

DESCRIPTION:

Destroy I/O staging communicator object iocomm.

INPUT PARAMETERS:

integer, intent(in) : : iocomm 


\subsubsection{2 zio_destroy_listentry}

INTERFACE:

integer function zio_destroy_listentry(name)

\section{DESCRIPTION:}

Remove the distributed array descriptor entry with variable name name from the descriptor list.

INPUT PARAMETERS:

character(len=*), intent(in) : : name

\subsubsection{3 zio_find_locnx}

INTERFACE:

integer function zio_find_locnx (nx,mype,totpes, locnx, begin)

DESCRIPTION:

Find local size locnx and zero-based offset begin for process mype when the global dimension size is $\mathrm{nx}$ and the total process count is totpes.

INPUT PARAMETERS:

integer, intent(in) $:: \mathrm{nx}$

integer, intent(in) : : mype

! global size

integer, intent(in) : : totpes

! PE rank

! total number of PEs

OUTPUT PARAMETERS:

integer, intent(out) : : locnx

integer, intent(out) : : begin

! local size for PE mype

! beginning index (zero-based)

\subsubsection{4 zio_get_comm}

INTERFACE:

integer function zio_get_comm(iocomm, comm)

DESCRIPTION:

Return the MPI communicator of I/O staging communicator object iocomm.

INPUT PARAMETERS:

integer, intent(in) : : iocomm

OUTPUT PARAMETERS:

integer, intent(out) : : comm 


\subsubsection{5 zio_get_coords}

INTERFACE:

integer function zio_get_coords(decomp, coords)

\section{DESCRIPTION:}

Returns the current process' coordinates in parallel decomposition object decomp.

INPUT PARAMETERS:

integer, intent(in) : : decomp

OUTPUT PARAMETERS:

integer, intent(out) : : coords $(:)$

\subsubsection{6 zio_get_distarray}

INTERFACE:

integer function zio_get_distarray (name, distarray)

DESCRIPTION:

Retrieves the distributed array descriptor for the variable with name name from the distributed array descriptor list.

INPUT PARAMETERS:

character(len=*), intent(in) : : name

OUTPUT PARAMETERS:

integer, intent(out) : : distarray

\subsubsection{7 zio_get_errtype}

INTERFACE:

integer function zio_get_errtype(errtype)

DESCRIPTION:

Returns ZioLib error type.

OUTPUT PARAMETERS:

integer, intent(out) : : errtype 


\subsubsection{8 zio_get_fileid}

INTERFACE:

integer function zio_get_fileid(filedesc,fileid)

\section{DESCRIPTION:}

Returns the file ID of file descriptor filedesc.

INPUT PARAMETERS:

integer, intent(in) : : filedesc

OUTPUT PARAMETERS:

integer, intent(out) : : fileid

\subsubsection{9 zio_get_ghstl}

INTERFACE:

integer function zio_get_ghstl(distarray,ghstl)

\section{DESCRIPTION:}

Returns the number of LHS ghost nodes (that is, ghost nodes preceding the real nodes) of distributed array descriptor distarray.

INPUT PARAMETERS:

integer, intent(in) : : distarray

OUTPUT PARAMETERS:

integer, intent(out) : : ghstl(*)

\subsubsection{0 zio_get_ghstr}

INTERFACE:

integer function zio_get_ghstr(distarray,ghstr)

\section{DESCRIPTION:}

Returns the number of RHS ghost nodes (that is, ghost nodes after the real nodes) of distributed array descriptor distarray.

INPUT PARAMETERS:

integer, intent(in) : : distarray

OUTPUT PARAMETERS:

integer, intent(out) : : ghstr(*) 


\subsubsection{1 zio_get_gsize}

INTERFACE:

integer function zio_get_gsize(distarray,gsize)

DESCRIPTION:

Returns the global grid sizes of distributed array descriptor distarray.

INPUT PARAMETERS:

integer, intent(in) : : distarray

OUTPUT PARAMETERS:

integer, intent(out) : : gsize(*)

\subsubsection{2 zio_get_index_order}

INTERFACE:

integer function zio_get_index_order(distarray, index_order)

DESCRIPTION:

Returns the array output index order of distributed array descriptor distarray.

INPUT PARAMETERS:

integer, intent(in) : : distarray

OUTPUT PARAMETERS:

integer, intent(out) : : index_order $(*)$

\subsubsection{3 zio_get_io_rank}

INTERFACE:

integer function zio_get_io_rank(iocomm,rank, io_rank)

DESCRIPTION:

Returns the process rank in I/O staging communicator object iocomm of process rank.

INPUT PARAMETERS:

integer, intent(in) : : iocomm

integer, intent(in) : : rank

OUTPUT PARAMETERS:

integer, intent (out) : : io_rank 


\subsubsection{4 zio_get_iocomm}

INTERFACE:

integer function zio_get_iocomm(filedesc,iocomm)

DESCRIPTION:

Returns I/O staging communicator object iocomm associated with file descriptor filedesc.

INPUT PARAMETERS:

integer, intent(in) : : filedesc

OUTPUT PARAMETERS:

integer, intent(out) : : iocomm

\subsubsection{5 zio_get_is_iope}

INTERFACE:

integer function zio_get_is_iope(iocomm,is_iope)

\section{DESCRIPTION:}

Returns a logical array indicating whether processes are I/O staging processes in I/O staging communicator object iocomm.

INPUT PARAMETERS:

integer, intent(in) :: iocomm

OUTPUT PARAMETERS:

logical, intent(out) : : is_iope $(0: *)$

\subsubsection{6 zio_get_loff}

INTERFACE:

integer function zio_get_loff(distarray,ndims,nblks, loff)

DESCRIPTION:

Returns the global grid offsets for all array dimensions for distributed array descriptor distarray on all processes. 
integer, intent(in) : : distarray

integer, intent(in) : : ndims

integer, intent(in) : : nblks

OUTPUT PARAMETERS:

integer, intent(out) : : loff(ndims,nblks, $0: *$ )

\subsubsection{7 zio_get_loff_me}

INTERFACE:

integer function zio_get_loff_me(distarray,ndims,loff)

DESCRIPTION:

Returns the global grid offsets for all array dimensions for distributed array descriptor distarray on the current process.

INPUT PARAMETERS:

integer, intent(in) : : distarray

integer, intent(in) : : ndims

OUTPUT PARAMETERS:

integer, intent(out) : : loff(ndims,*)

\subsubsection{8 zio_get_lsize}

INTERFACE:

integer function zio_get_lsize(distarray,ndims,nblks,lsize)

DESCRIPTION:

Returns the local grid sizes for all array dimensions for distributed array descriptor distarray on all processes.

INPUT PARAMETERS:

integer, intent(in) : : distarray

integer, intent(in) : : ndims

integer, intent(in) : : nblks

OUTPUT PARAMETERS:

integer, intent(out) :: lsize(ndims,nblks,0:*) 


\subsubsection{9 zio_get_lsize_me}

INTERFACE:

integer function zio_get_lsize_me(distarray,ndims,lsize)

\section{DESCRIPTION:}

Returns the local grid sizes for all array dimensions for distributed array descriptor distarray on the current process.

INPUT PARAMETERS:

integer, intent(in) : : distarray

integer, intent(in) : : ndims

OUTPUT PARAMETERS:

integer, intent(out) : : lsize(ndims,*)

\subsubsection{0 zio_get_me}

INTERFACE:

integer function zio_get_me(iocomm,me)

DESCRIPTION:

Returns the process rank in the I/O staging communicator associated with the object iocomm.

INPUT PARAMETERS:

integer, intent(in) : : iocomm

OUTPUT PARAMETERS:

integer, intent(out) : : me

\subsubsection{1 zio_get_method}

INTERFACE:

integer function zio_get_method(filedesc,method)

\section{DESCRIPTION:}

Returns the I/O method (netCDF, direct-access or sequential-access?) for file descriptor filedesc. 
integer, intent(in) : : filedesc

OUTPUT PARAMETERS:

integer, intent(out) : : method

\subsubsection{2 zio_get_nblks}

INTERFACE:

integer function zio_get_nblks (distarray,nblks)

DESCRIPTION:

Returns the number of disjoint rectangular data blocks of distributed array descriptor distarray.

INPUT PARAMETERS:

integer, intent(in) : : distarray

OUTPUT PARAMETERS:

integer, intent(out) : : nblks $(0: *)$

\subsubsection{3 zio_get_nblks_max}

INTERFACE:

integer function zio_get_nblks_max(distarray,nblks)

DESCRIPTION:

Returns the maximim number of disjoint rectangular data blocks of distributed array descriptor distarray over PEs. Size of the 2 nd dimension of $\operatorname{loff}(:,:,:)$ and $\operatorname{lsize}(:,:,:)$ of distributed array desc objects.

INPUT PARAMETERS:

integer, intent(in) : : distarray

OUTPUT PARAMETERS:

integer, intent(out) : : nblks 


\subsubsection{4 zio_get_ndims_decomp}

INTERFACE:

integer function zio_get_ndims_decomp(decomp,ndims)

DESCRIPTION:

Returns the number of dimensions of parallel decomposition object decomp.

INPUT PARAMETERS:

integer, intent(in) : : decomp

OUTPUT PARAMETERS:

integer, intent(out) : : ndims

\subsubsection{5 zio_get_ndims_distarray}

INTERFACE:

integer function zio_get_ndims_distarray(distarray,ndims)

DESCRIPTION:

Returns the number of dimensions for distributed arrays associated with descriptor distarray.

INPUT PARAMETERS:

integer, intent(in) : : distarray

OUTPUT PARAMETERS:

integer, intent(out) : : ndims

\subsubsection{6 zio_get_ndims_par}

INTERFACE:

integer function zio_get_ndims_par(filedesc,ndims_par)

DESCRIPTION:

Returns the minimum number of array dimensions for parallel I/O for the file associated with descriptor filedesc.

INPUT PARAMETERS:

integer, intent(in) : : filedesc

OUTPUT PARAMETERS:

integer, intent(out) : : ndims_par 


\subsubsection{7 zio_get_npes}

INTERFACE:

integer function zio_get_npes(decomp,npes)

\section{DESCRIPTION:}

Returns the numbers of processes for parallel decomposition descriptor decomp.

INPUT PARAMETERS:

integer, intent(in) : : decomp

OUTPUT PARAMETERS:

integer, intent(out) : : npes(*)

\subsubsection{8 zio_get_niopes}

INTERFACE:

integer function zio_get_niopes(iocomm,niopes)

\section{DESCRIPTION:}

Returns the number of I/O staging processes for the staging communicator associated with iocomm.

INPUT PARAMETERS:

integer, intent(in) : : iocomm

OUTPUT PARAMETERS:

integer, intent(out) : : niopes

\subsubsection{9 zio_get_ranks_cmp}

INTERFACE:

integer function zio_get_ranks_cmp(iocomm,ranks_cmp)

DESCRIPTION:

Returns the process ranks in the compute communicator, of the I/O staging processes in staging communicator object iocomm.

INPUT PARAMETERS:

integer, intent(in) : : iocomm

OUTPUT PARAMETERS:

integer, intent(out) : : ranks_cmp $(0: *)$ 


\subsubsection{0 zio_get_reclen}

INTERFACE:

integer function zio_get_reclen(filedesc,reclen)

\section{DESCRIPTION:}

Returns the record length of the file associated with file descriptor filedesc.

INPUT PARAMETERS:

integer, intent(in) : : filedesc

OUTPUT PARAMETERS:

integer, intent(out) : : reclen

\subsubsection{1 zio_get_zdim_size}

INTERFACE:

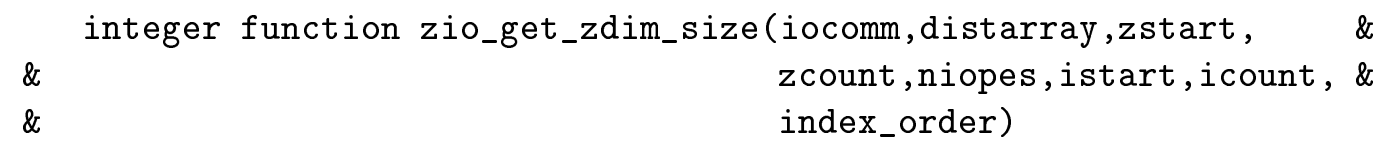

DESCRIPTION:

Returns "Z" dimension's offset and size on an I/O staging process. A zero is returned for $z$ count if the current process is not an $\mathrm{I} / \mathrm{O}$ staging process.

INPUT PARAMETERS:

integer, intent(in) : : iocomm, distarray

integer, intent(in), optional : : niopes

integer, intent(in), optional : : istart(:), icount(:)

integer, intent(in), optional : : index_order(:)

OUTPUT PARAMETERS:

integer, intent(out) : : zstart, zcount

\subsubsection{2 zio_get_zio_comm}

INTERFACE:

integer function zio_get_zio_comm(comm) 
DESCRIPTION:

Returns the compute communicator.

OUTPUT PARAMETERS:

integer, intent(out) : : comm

\subsubsection{3 zio_get_zio_initialized}

INTERFACE:

integer function zio_get_zio_initialized(initialized)

DESCRIPTION:

Checks whether ZioLib is initialized.

OUTPUT PARAMETERS:

logical, intent(out) : : initialized

\subsubsection{4 zio_get_zio_me}

INTERFACE:

integer function zio_get_zio_me(me)

DESCRIPTION:

Returns the process rank in the compute communicator.

OUTPUT PARAMETERS:

integer, intent(out) : : me

\subsubsection{5 zio_get_zio_npes}

INTERFACE:

integer function zio_get_zio_npes(npes)

DESCRIPTION:

Returns the number of processes in the compute communicator.

OUTPUT PARAMETERS:

integer, intent(out) : : npes 


\subsubsection{6 zio_new_decomp}

INTERFACE:

integer function zio_new_decomp(decomp,p1,p2,p3,p4,p5)

\section{DESCRIPTION:}

Create a descriptor for parallel decomposition of up to 5-D which has $\mathrm{p} 1, \mathrm{p} 2, \ldots$ processes along axes. Here, process ranks change fastest along the first axis, then second axis,... (In MPI's Cartesian process topology, rank ordering is the opposite.)

OUTPUT PARAMETERS:

integer, intent(out) : : decomp

INPUT PARAMETERS:

integer, intent(in) $:: \mathrm{p} 1$
integer, optional, intent(in) $:: \mathrm{p} 2$
integer, optional, intent(in) $:: \mathrm{p} 3$
integer, optional, intent(in) $:: \mathrm{p} 4$
integer, optional, intent(in) $:: \mathrm{p} 5$

\subsubsection{7 zio_new_distarray}

INTERFACE:

$$
\begin{aligned}
& \text { integer function zio_new_distarray(distarray,nblks,ndims,loff, \& } \\
& \& \\
& \text { lsize,ghstl,ghstr, index_order) }
\end{aligned}
$$

\section{DESCRIPTION:}

Create a descriptor for a distributed array made of nblks disjoint rectangular blocks of ndims dimensions whose offsets, local sizes, LHS ghost nodes, RHS ghost nodes and the output order are given respectively by loff (1:ndims, $1: \mathrm{nblks})$, 1 size(1:ndims, $1: \mathrm{nblks})$, ghstl (1:ndims), ghstr (1:ndims), and index_order (1:ndims).

OUTPUT PARAMETERS:

integer, intent(out) : : distarray ! dist array obj

INPUT PARAMETERS:

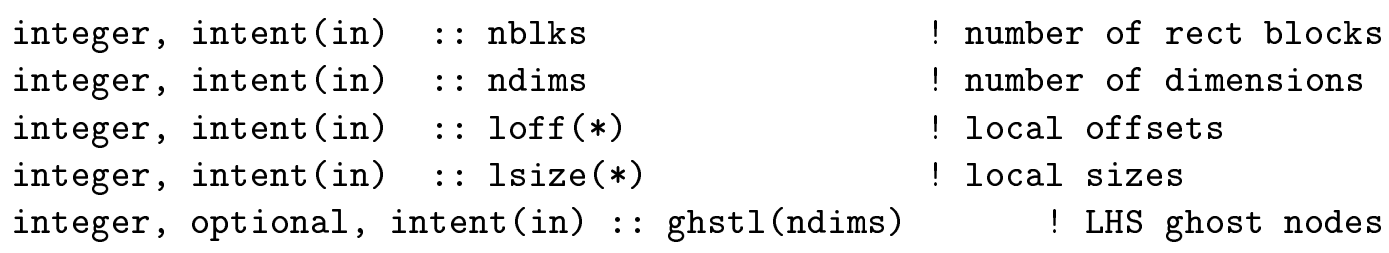




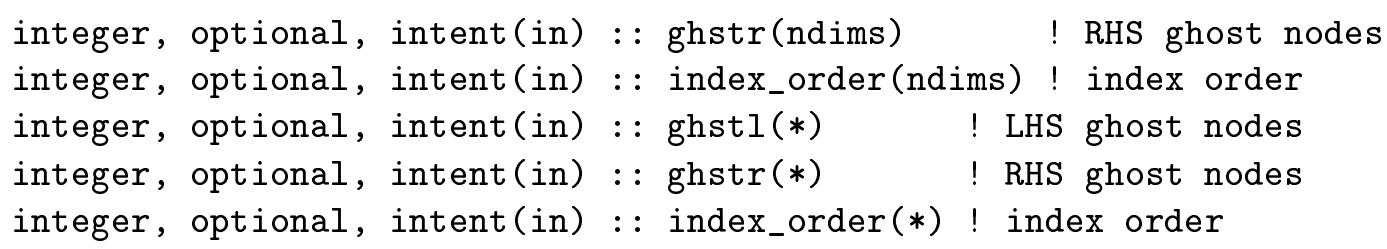

\subsubsection{8 zio_define_distarray}

INTERFACE:

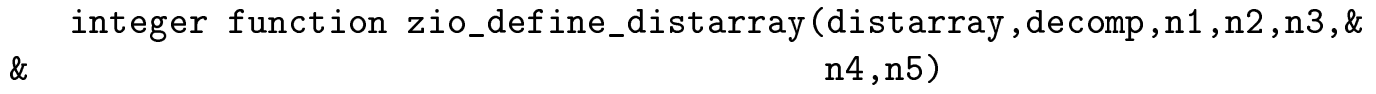

\section{DESCRIPTION:}

Create a descriptor for the distributed array of grid sizes $\mathrm{n} 1, \mathrm{n} 2, \ldots$ for the parallel decomposition (up to 5-D) denoted by decomp. If a grid size is not evenly divisible by the respective process count, the excess grid points (that is, remainder) are evenly distributed among the lowest-rank processes along the dimension.

OUTPUT PARAMETERS:

integer, intent(out) : : distarray

INPUT PARAMETERS:

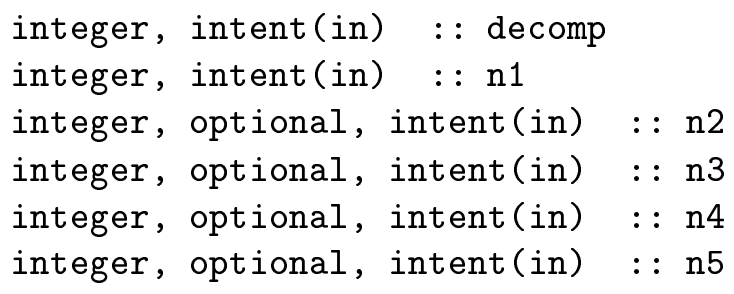

\subsubsection{9 zio_import_distarray}

INTERFACE:

integer function zio_import_distarray(distarray, user_func)

\section{DESCRIPTION:}

Create a distributed array descriptor whose distributed grid is defined in user-provided function, user func.

OUTPUT PARAMETERS:

integer, intent(out) : : distarray 
INPUT PARAMETERS:

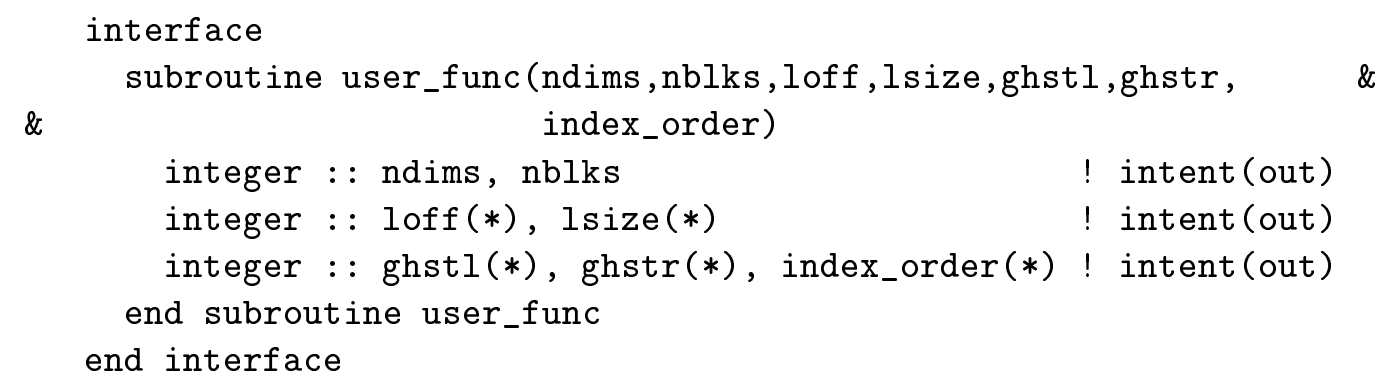

\subsubsection{0 zio_new_iocomm}

\section{INTERFACE:}

integer function zio_new_iocomm(iocomm,niopes)

\section{DESCRIPTION:}

Create an I/O staging communicator object with a staging communicator made of the first niopes processes.

OUTPUT PARAMETERS:

integer, intent(out) : : iocomm ! communicator obj ID

INPUT PARAMETERS:

integer, intent(in) : : niopes $\quad$ \# of I/O staging processes

\subsubsection{1 zio_new_iocomm_incl}

\section{INTERFACE:}

integer function zio_new_iocomm_incl(iocomm,n,ranks)

\section{DESCRIPTION:}

Create an I/O staging communicator object with a staging communicator made of the processes whose ranks are specified by $\operatorname{ranks}(1: n)$.

\section{OUTPUT PARAMETERS:}

integer, intent(out) : : iocomm ! communicator obj ID

INPUT PARAMETERS:

integer, intent(in) :: $\mathrm{n} \quad$ ! \# of I/O staging processes in comm

integer, intent(in) : $\operatorname{ranks}(n) \quad$ ! ranks 


\subsubsection{2 zio_new_iocomm_member}

INTERFACE:

integer function zio_new_iocomm_member(iocomm, is_iope)

DESCRIPTION:

Create an I/O staging communicator object with a staging communicator made of the processes with is_iope $=$.true.

OUTPUT PARAMETERS:

integer, intent(out) : : iocomm ! communicator obj ID

INPUT PARAMETERS:

logical, intent(in) : : is_iope ! belongs to staging comm?

\subsubsection{3 zio_set_ghostlayers}

INTERFACE:

integer function zio_set_ghostlayers(distarray,ghstl,ghstr)

DESCRIPTION:

Sets ghost nodes for distributed array descriptor distarray.

INPUT PARAMETERS:

integer, intent(in) : ! distarray array distribution

integer, intent(in) : : ghstl(:), ghstr(:) ! LHS and RHS ghost nodes

\subsubsection{4 zio_set_index_order}

INTERFACE:

integer function zio_set_index_order(distarray, index_order)

DESCRIPTION:

Set output index order for distributed array descriptor distarray.

INPUT PARAMETERS:

integer, intent(in) : distarray ! array distribution

integer, intent(in) : : index_order(:) ! index order 


\subsubsection{5 zio_set_ndims_par}

INTERFACE:

integer function zio_set_ndims_par(filedesc,ndims_par)

\section{DESCRIPTION:}

Set the parameter for the minumum number of array dimensions for parallel I/O.

INPUT PARAMETERS:

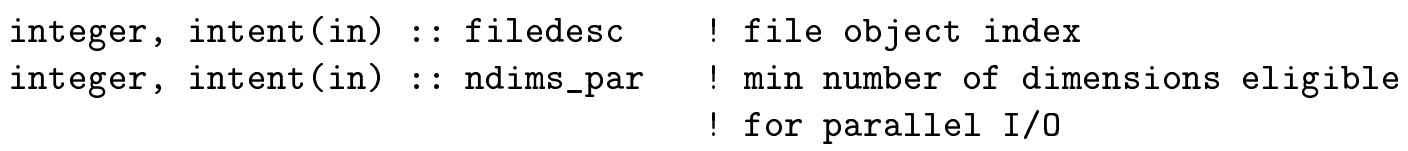

\subsection{Module zio_remap (Source File: zio_remap.F90)}

Module zio_remap provides procedures for remapping from and to I/O staging processes.

USES:

use zio_data

PUBLIC MEMBER FUNCTIONS:

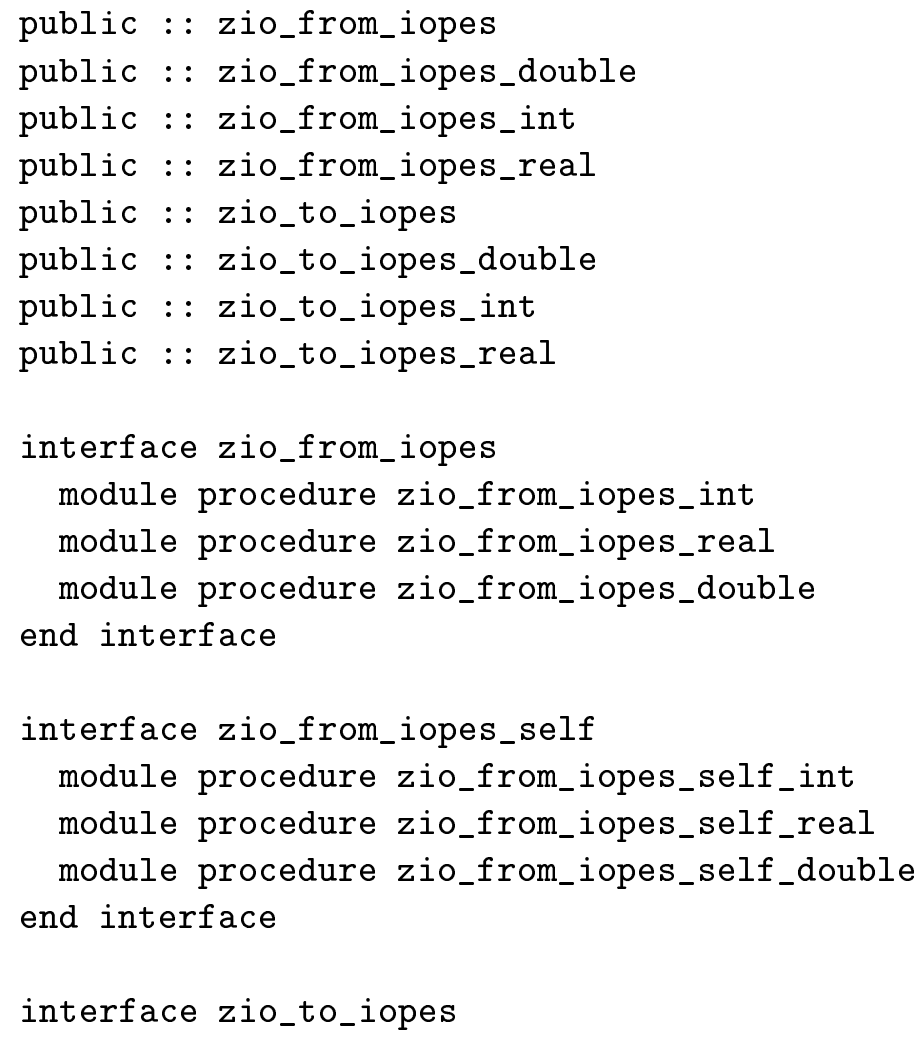




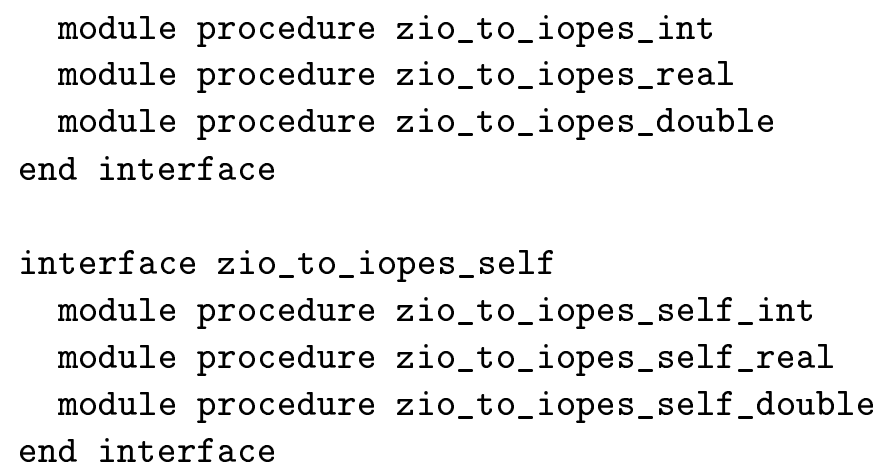

\subsection{1 zio_from_iopes_double}

INTERFACE:

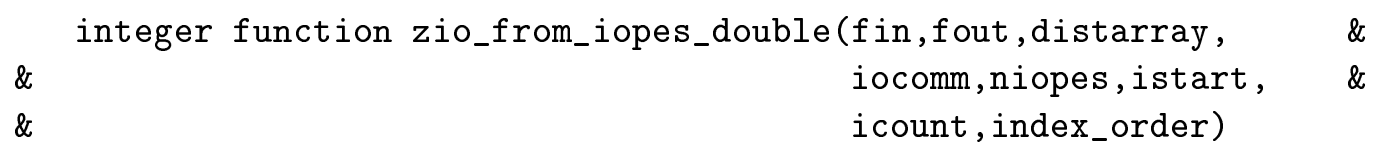

DESCRIPTION:

Construct distributed arrays from Z-decomposed arrays on I/O staging PEs

INPUT PARAMETERS:

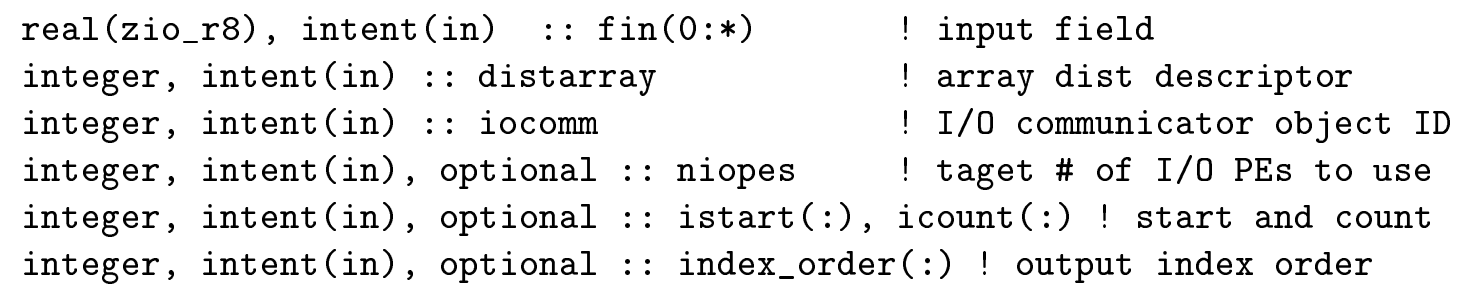

\subsection{2 zio_from_iopes_int}

INTERFACE:

$\begin{array}{lcr} & \text { integer function zio_from_iopes_int(fin,fout, distarray, } & \text { \& } \\ \& & \text { iocomm,niopes, istart, } & \text { \& } \\ \& & \text { icount, index_order) } & \end{array}$

DESCRIPTION:

Construct distributed arrays from Z-decomposed arrays on I/O staging PEs 


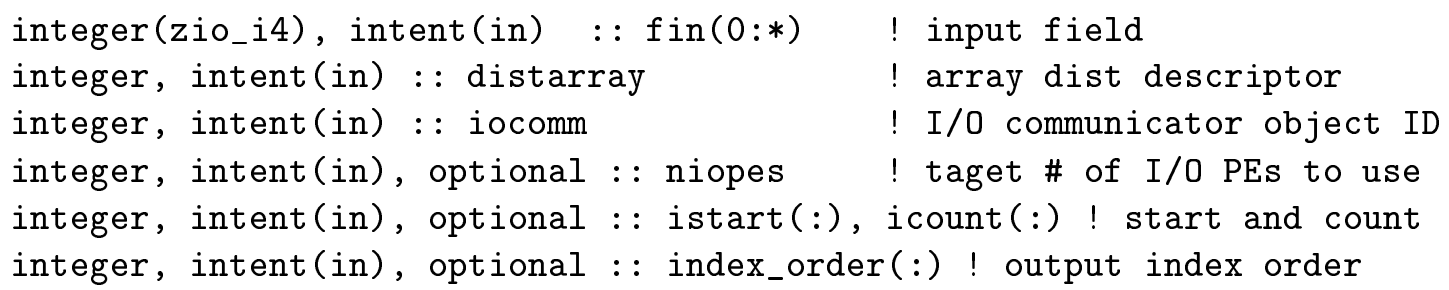

\subsection{3 zio_from_iopes_real}

INTERFACE:

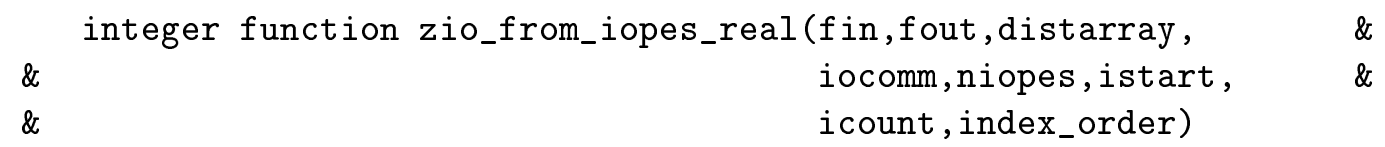

DESCRIPTION:

Construct distributed arrays from Z-decomposed arrays on I/O staging PEs

INPUT PARAMETERS:

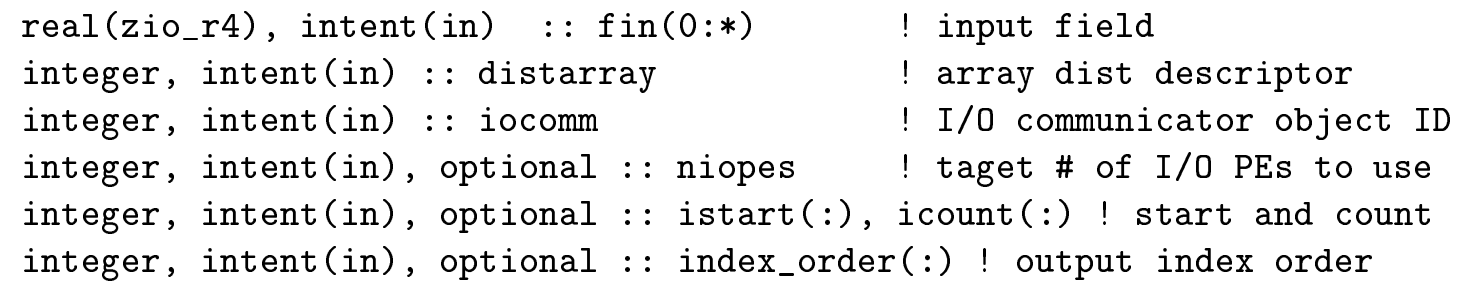

OUTPUT PARAMETERS:

real(zio_r4), intent(out) : : fout $(0: *) \quad$ ! remapped array

\subsection{4 zio_to_iopes_double}

INTERFACE:

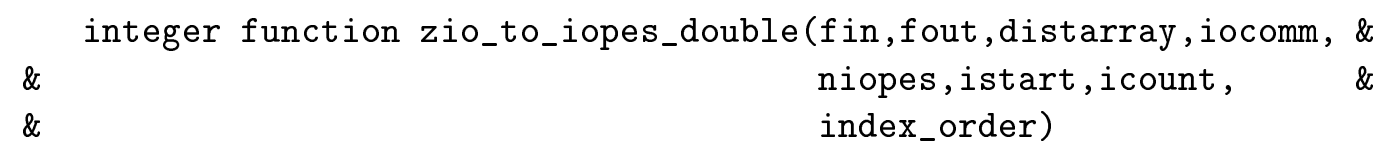

DESCRIPTION:

Remaps distributed local arrays to Z-decomposed arrays on I/O staging PEs 


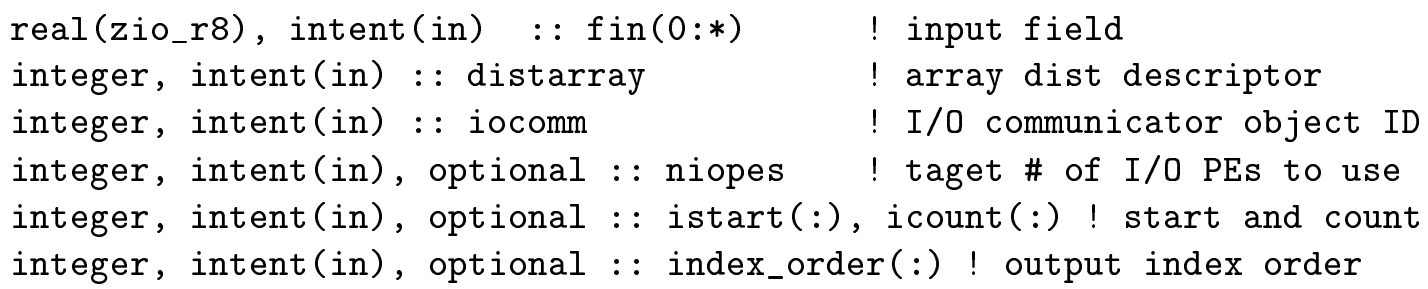

OUTPUT PARAMETERS:

real(zio_r8), intent(out) : : fout $(0: *) \quad$ ! remapped array

\subsection{5 zio_to_iopes_int}

INTERFACE:

integer function zio_to_iopes_int(fin,fout,distarray, iocomm, \&

$\begin{array}{ll}\& & \text { niopes, istart, icount, \& } \\ \& & \text { index_order) }\end{array}$

\section{DESCRIPTION:}

Remaps distributed local arrays to Z-decomposed arrays on I/O staging PEs

INPUT PARAMETERS:

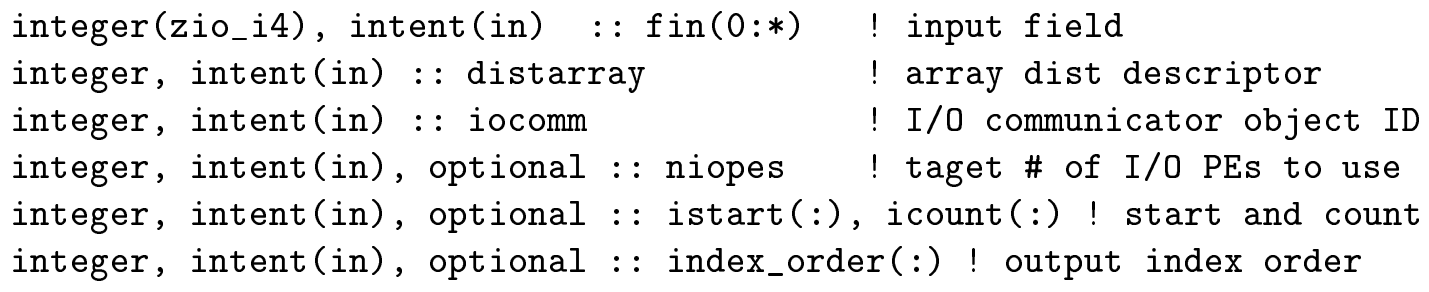

OUTPUT PARAMETERS:

integer(zio_i4), intent(out) : fout $(0: *)$ ! remapped array

\subsection{6 zio_to_iopes_real}

INTERFACE:

$\begin{array}{ccc} & \text { integer function zio_to_iopes_real (fin,fout,distarray, iocomm, } & \& \\ \& & \text { niopes,istart,icount, } & \text { \& } \\ \& & \text { index_order) } & \end{array}$

DESCRIPTION:

Remaps distributed local arrays to Z-decomposed arrays on I/O staging PEs 


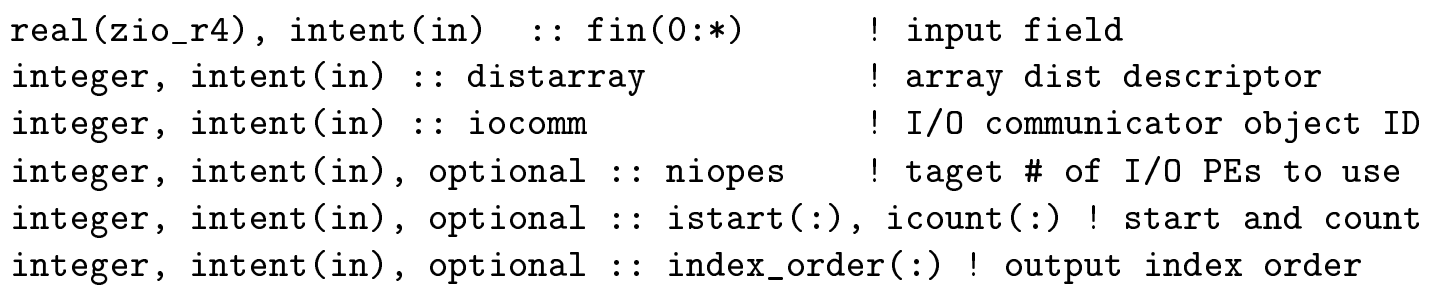

\subsection{Module zio_binary (Source File: zio_binary.F90)}

Module zio_binary provides procedures for Fortran direct- and sequential-access unformatted I/O.

INTERFACE:

module zio_binary

USES:

use zio_data

use zio_remap

PUBLIC MEMBER FUNCTIONS:

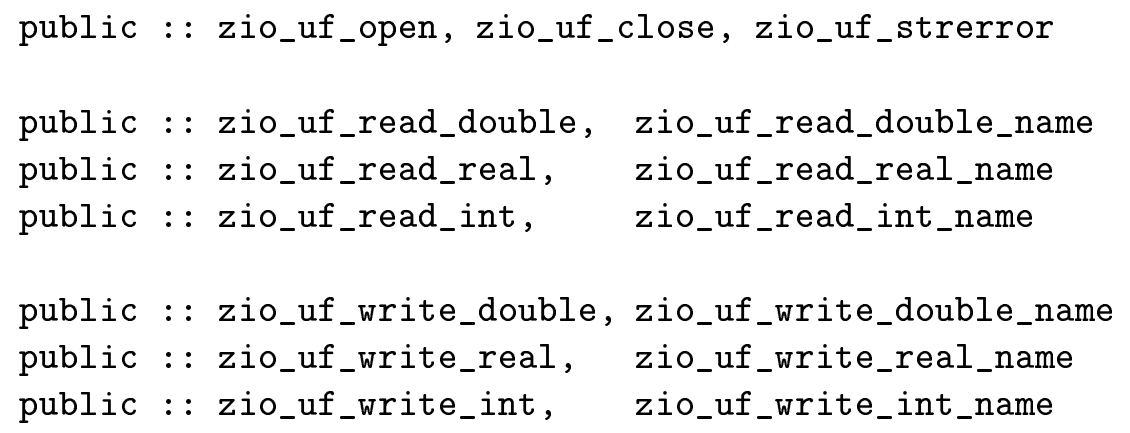

\subsection{1 zio_uf_close}

INTERFACE:

integer function zio_uf_close(filedesc,status)

\section{DESCRIPTION:}

Close a file

INPUT PARAMETERS:

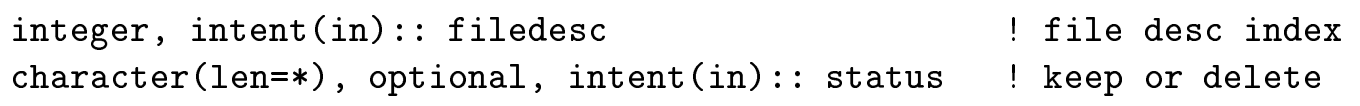




\subsection{2 zio_uf_open}

INTERFACE:

$$
\begin{array}{r}
\text { integer function zio_uf_open(filedesc,iocomm, unit,file, } \\
\text { status, access,recl,position, action) }
\end{array}
$$

DESCRIPTION:

Open a file for reading and/or writing

OUTPUT PARAMETERS:

integer, intent(out): filedesc $\quad$ file desc index

INPUT PARAMETERS:

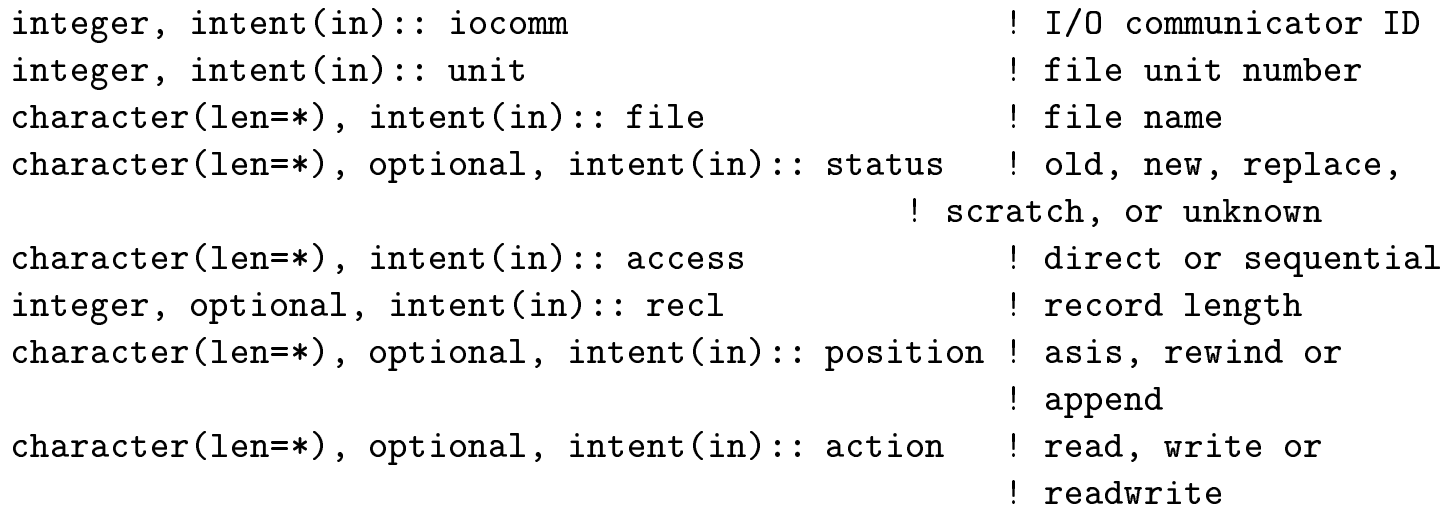

\subsection{3 zio_uf_read_double}

INTERFACE:

$$
\begin{gathered}
\text { integer function zio_uf_read_double(filedesc,distarray, arr, rec, \& } \\
\text { istart, icount, } \mathrm{n})
\end{gathered}
$$

DESCRIPTION:

Unformatted read

INPUT PARAMETERS:

integer, intent(in): : filedesc, distarray

integer, optional, intent(in) : rec ! global beg record number

! (1-based)

integer, optional, intent(in) : : istart(:), icount(:)

integer, optional, intent(in) : : $\mathrm{n} \quad$ ! \# of elements in case of

OUTPUT PARAMETERS:

! replicated array

real(zio_r8), intent(out) : : $\operatorname{arr}(*)$ 


\subsection{4 zio_uf_read_double_name}

INTERFACE:

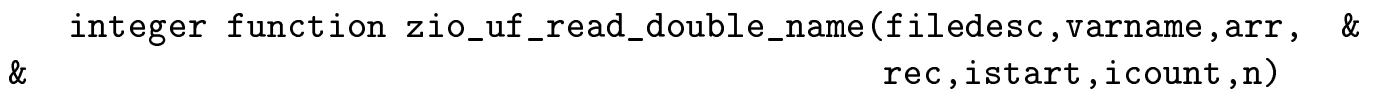

\section{DESCRIPTION:}

Unformatted read for variable with variable name of 'varname'. The distributed array descriptor for the variable must have been added to the descriptor list with the name of 'varname'. See zio_add_to_list.

INPUT PARAMETERS:

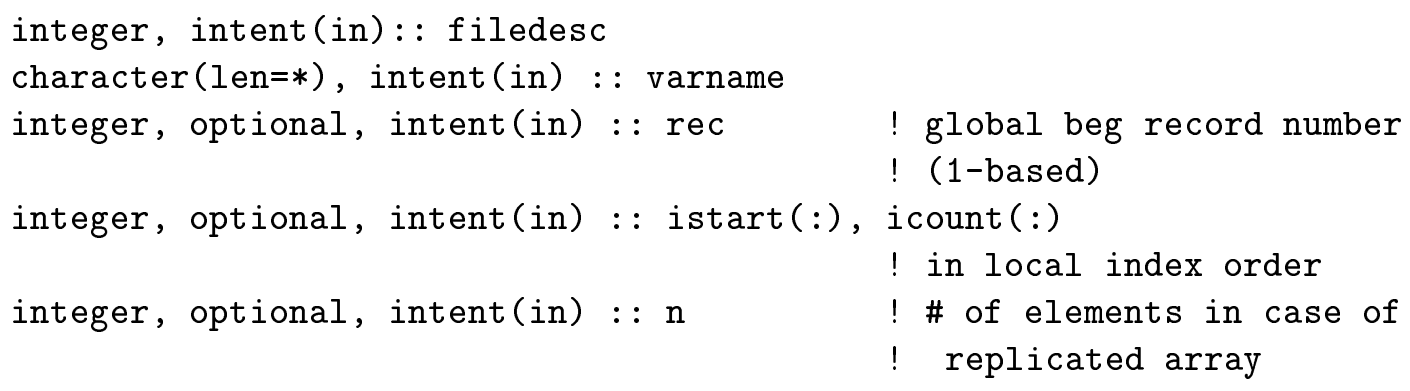

OUTPUT PARAMETERS:

real(zio_r8), intent(out) : : $\operatorname{arr}(*)$

\subsection{5 zio_uf_read_int}

INTERFACE:

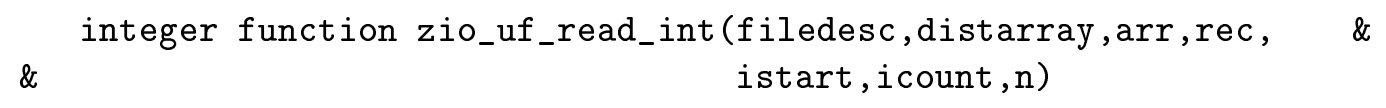

\section{DESCRIPTION:}

Unformatted read

INPUT PARAMETERS:

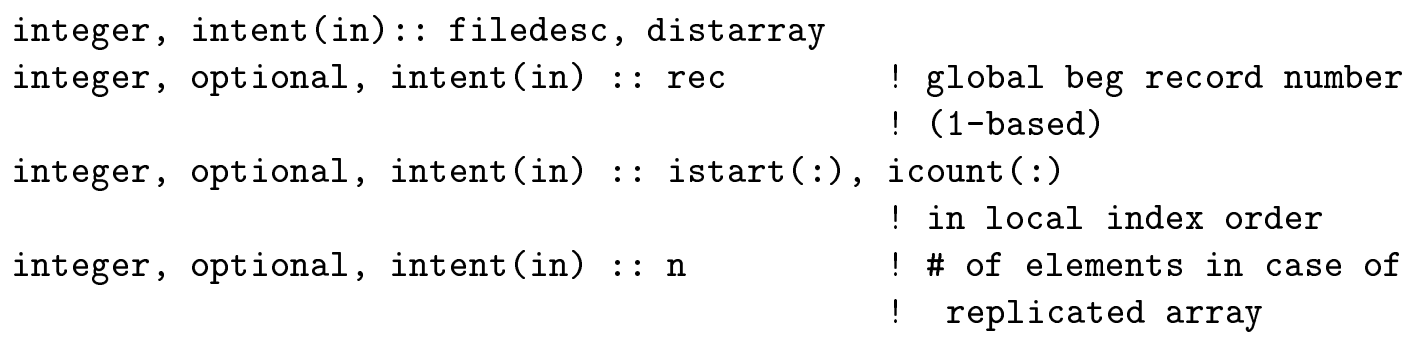

OUTPUT PARAMETERS:

integer(zio_i4), intent(out) : : $\operatorname{arr}(*)$ 


\subsection{6 zio_uf_read_int_name}

INTERFACE:

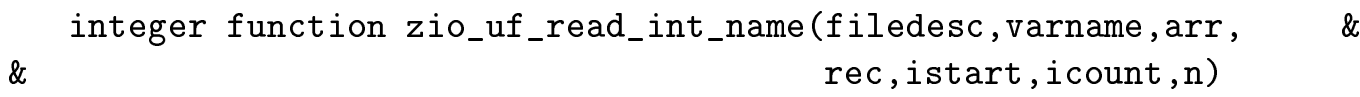

\section{DESCRIPTION:}

Unformatted read for variable with variable name of 'varname'. The distributed array descriptor for the variable must have been added to the descriptor list with the name of 'varname'. See zio_add_to_list.

INPUT PARAMETERS:

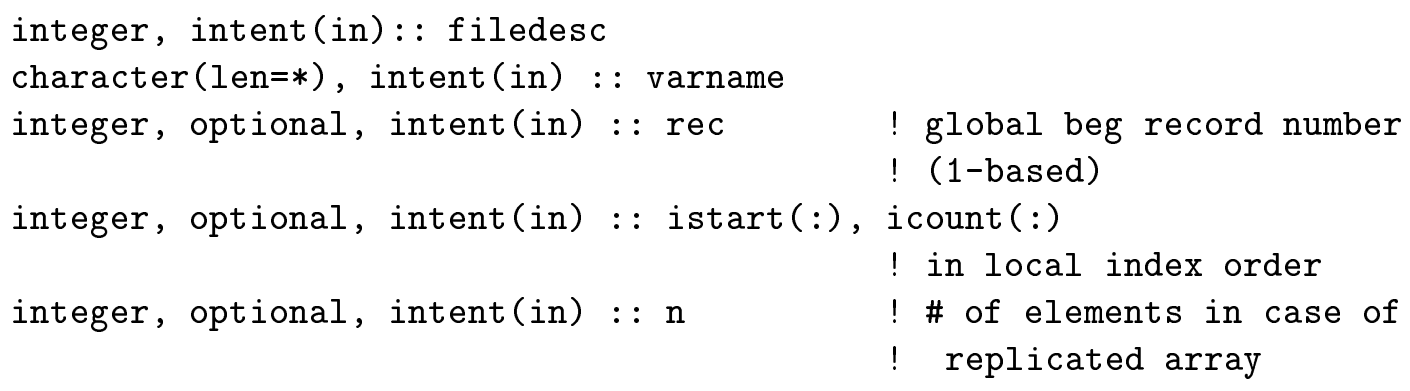

OUTPUT PARAMETERS:

integer(zio_i4), intent(out) : : $\operatorname{arr}(*)$

\subsection{7 zio_uf_read_real}

INTERFACE:

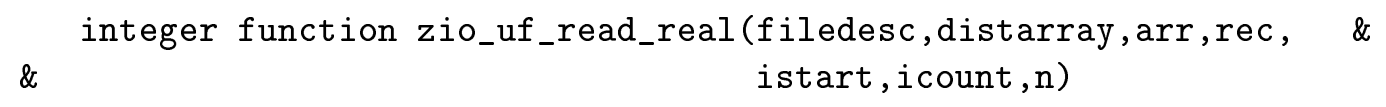

\section{DESCRIPTION:}

Unformatted read

INPUT PARAMETERS:

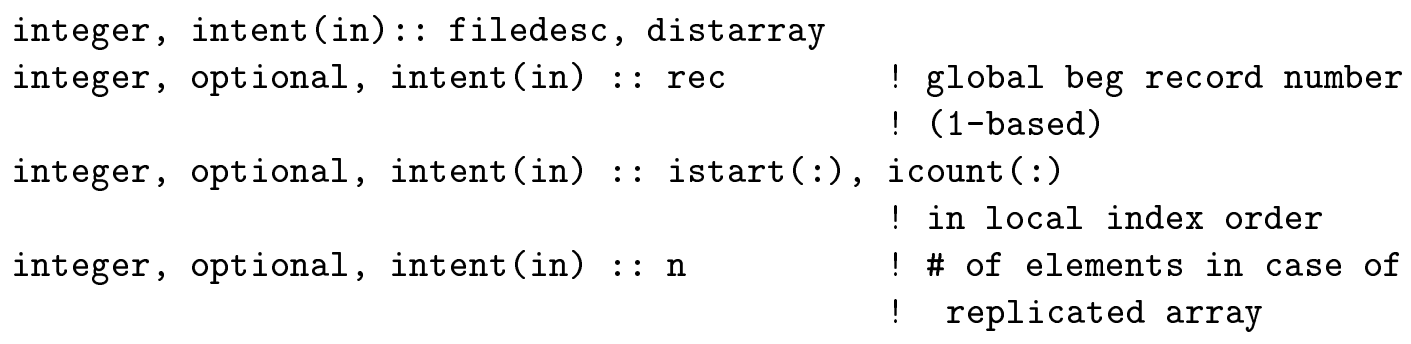

OUTPUT PARAMETERS:

real(zio_r4), intent(out) : : $\operatorname{arr}(*)$ 


\subsection{8 zio_uf_read_real_name}

INTERFACE:

$$
\begin{aligned}
& \text { integer function zio_uf_read_real_name(filedesc,varname,arr, \& } \\
& \& \\
& \text { rec, istart, icount, } n \text { ) }
\end{aligned}
$$

\section{DESCRIPTION:}

Unformatted read for variable with variable name of 'varname'. The distributed array descriptor for the variable must have been added to the descriptor list with the name of 'varname'. See zio_add_to_list.

INPUT PARAMETERS:

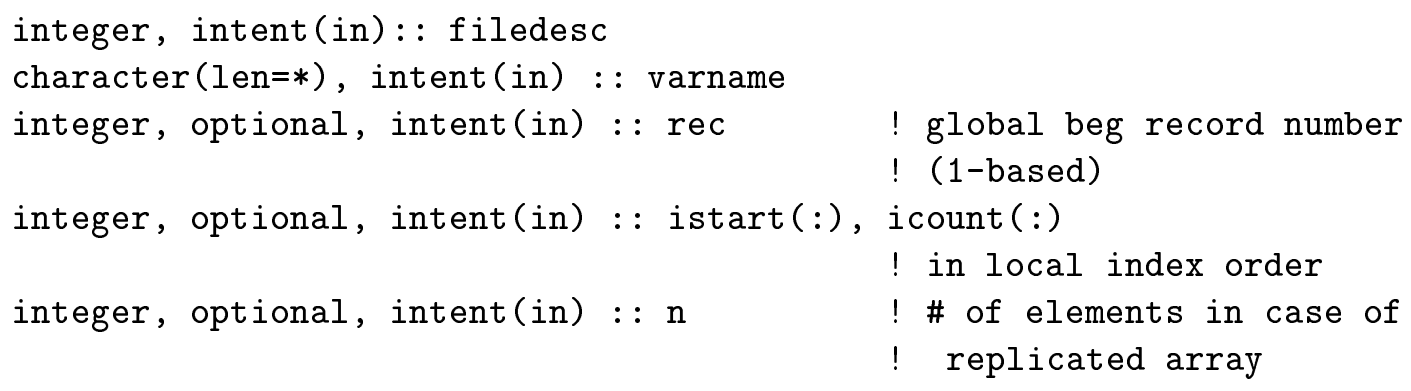

OUTPUT PARAMETERS:

real(zio_r4), intent(out) : : $\operatorname{arr}(*)$

\subsection{9 zio_uf_strerror}

INTERFACE:

function zio_uf_strerror(errcode)

DESCRIPTION:

Returns an error message string of len=80 for a unformatted I/O error given by errcode.

INPUT PARAMETERS:

integer, intent(in) : : errcode

OUTPUT PARAMETERS:

character(len=80) : : zio_uf_strerror 


\subsubsection{0 zio_uf_write_double}

INTERFACE:

$$
\begin{array}{r}
\text { integer function zio_uf_write_double(filedesc, distarray, arr, } \\
\text { rec,istart,icount, } n)
\end{array}
$$

\section{DESCRIPTION:}

Unformatted write

INPUT PARAMETERS:

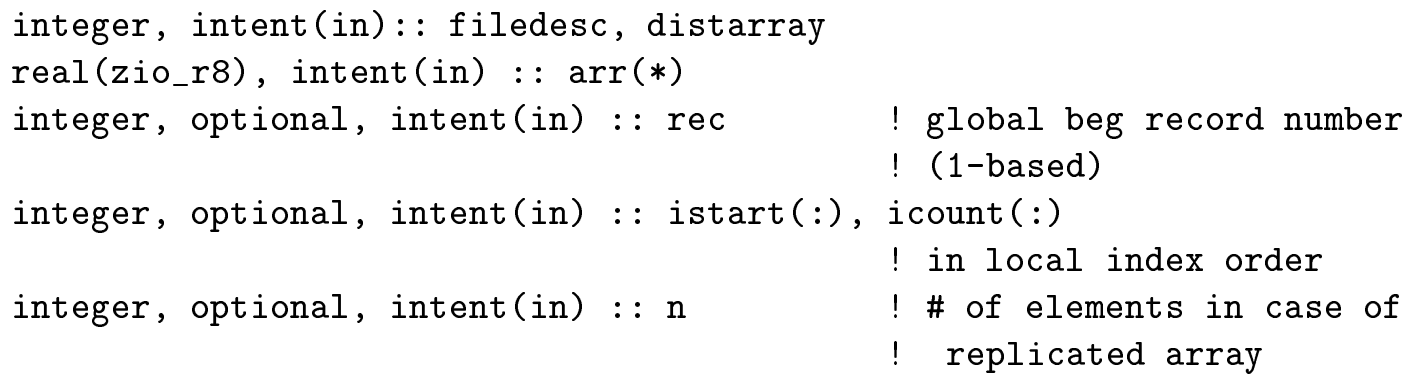

\subsubsection{1 zio_uf_write_double_name}

\section{INTERFACE:}

$$
\&
$$

integer function zio_uf_write_double_name(filedesc,varname,arr, \&

\section{DESCRIPTION:}

Unformatted write for variable with variable name of 'varname'. The distributed array descriptor for the variable must have been added to the descriptor list with the name of 'varname'. See zio_add_to_list.

INPUT PARAMETERS:

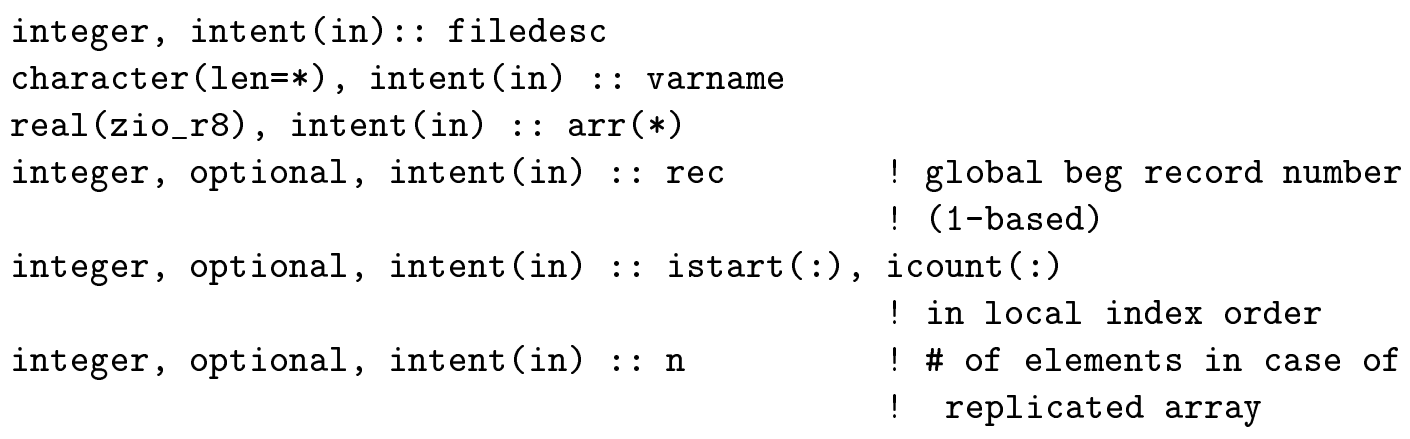




\subsubsection{2 zio_uf_write_int}

INTERFACE:

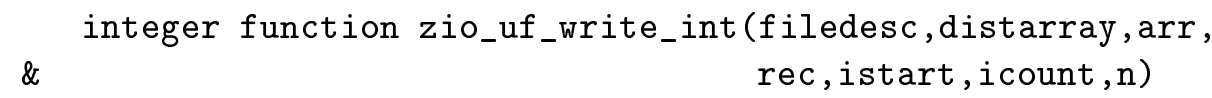

$\&$

DESCRIPTION:

Unformatted write

INPUT PARAMETERS:

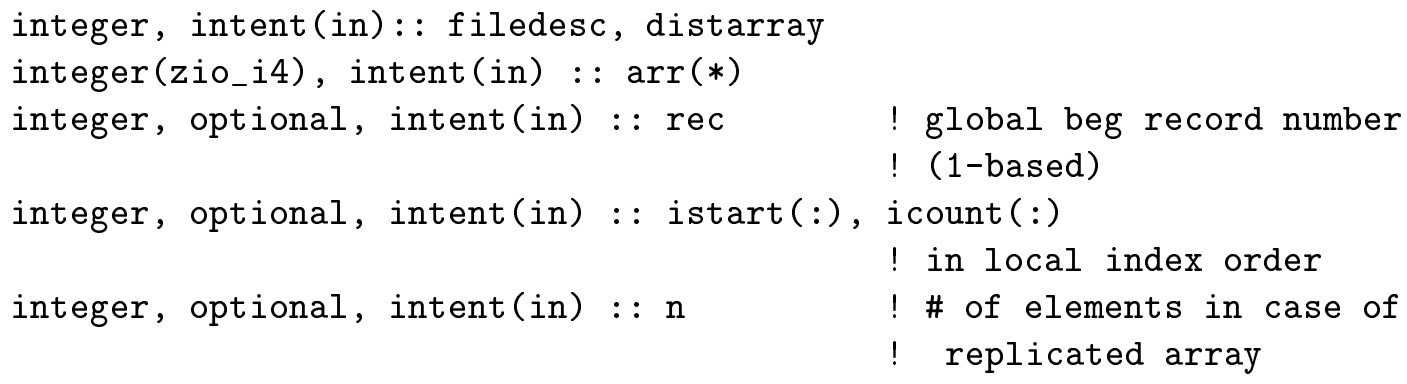

\subsubsection{3 zio_uf_write_int_name}

\section{INTERFACE:}

$$
\text { \& }
$$$$
\text { integer function zio_uf_write_int_name(filedesc,varname,arr, \& }
$$

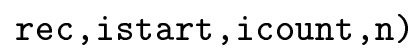

\section{DESCRIPTION:}

Unformatted write for variable with variable name of 'varname'. The distributed array descriptor for the variable must have been added to the descriptor list with the name of 'varname'. See zio_add_to_list.

INPUT PARAMETERS:

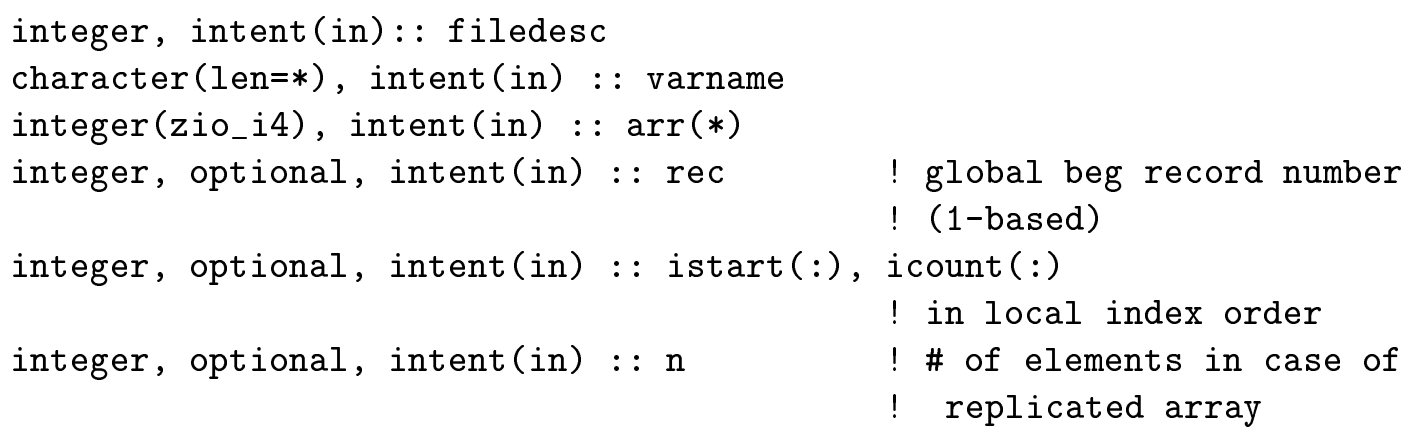




\subsubsection{4 zio_uf_write_real}

INTERFACE:

$$
\begin{array}{r}
\text { integer function zio_uf_write_real (filedesc,distarray, arr, } \\
\text { rec,istart,icount, } \mathrm{n})
\end{array} \quad \text { \& }
$$

\section{DESCRIPTION:}

Unformatted write

INPUT PARAMETERS:

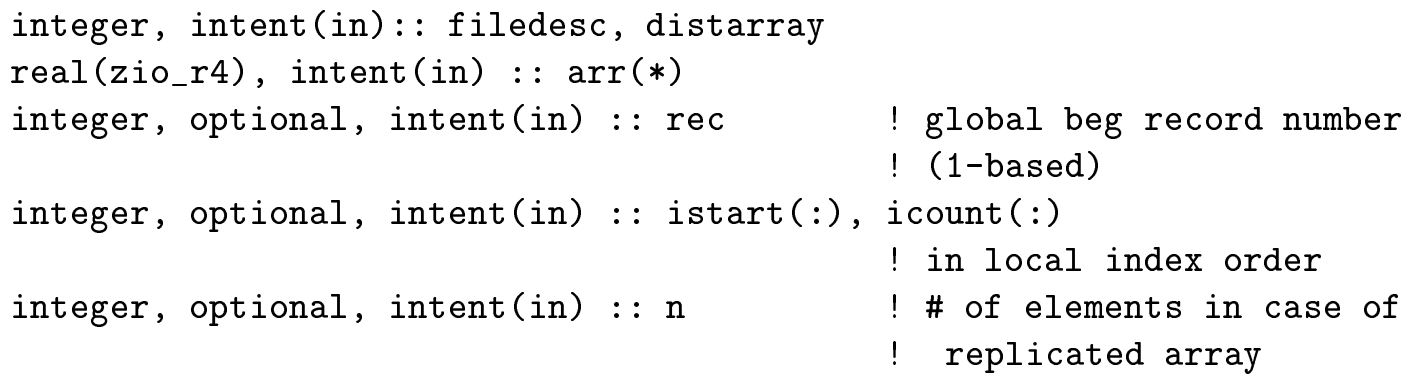

\subsubsection{5 zio_uf_write_real_name}

\section{INTERFACE:}

$$
\&
$$$$
\text { integer function zio_uf_write_real_name(filedesc,varname,arr, \& }
$$$$
\text { rec, istart, icount, } \mathrm{n} \text { ) }
$$

\section{DESCRIPTION:}

Unformatted write for variable with variable name of 'varname'. The distributed array descriptor for the variable must have been added to the descriptor list with the name of 'varname'. See zio_add_to_list.

INPUT PARAMETERS:

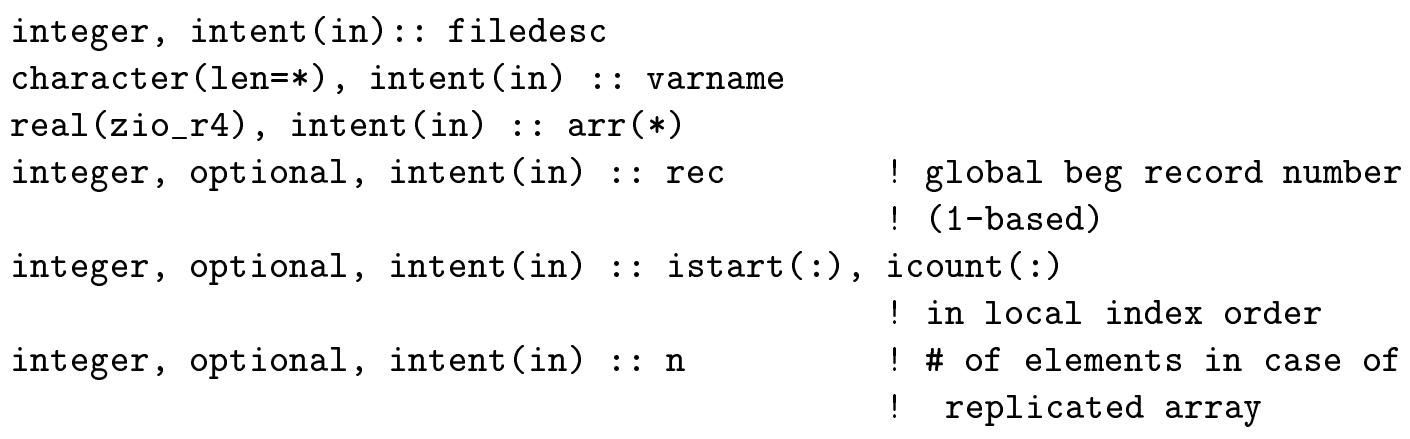




\subsection{Module zio_netcdf77 (Source File: zio_netcdf77.F90)}

Module zio_netcdf77 is a collection of wrappers for routines of the netCDF Fortran 77 interface.

INTERFACE:

module zio_netcdf77

USES:

use zio_data

use zio_remap

\#include <netcdf.inc>

\subsection{1 zio_nf__comm}

INTERFACE:

integer function zio_nf_-_comm(iocomm)

\section{DESCRIPTION:}

Sets the communicator for netCDF I/O to the communicator associated with I/O staging communicator object iocomm. Specific to the parallel netCDF library being developed at NERSC.

INPUT PARAMETERS:

$$
\text { integer, intent(in) : : iocomm ! I/O staging comm object }
$$

\subsection{2 zio_nf__create}

INTERFACE:

integer function zio_nf_-create (path,cmode, initialsize,
chunksize, iocomm,filedesc)

\section{DESCRIPTION:}

Creates a netCDF file using I/O staging communicator object iocomm. Returns the created file descriptor in filedesc. Specific to the parallel netCDF library being developed at NERSC.

INPUT PARAMETERS: 


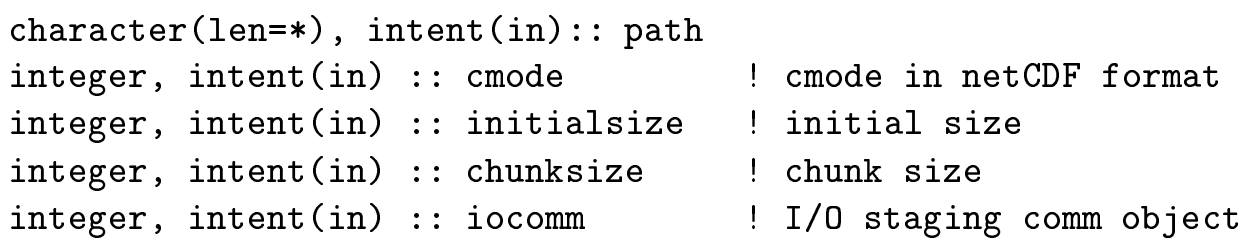

\subsection{3 zio_nf__create_mp}

INTERFACE:

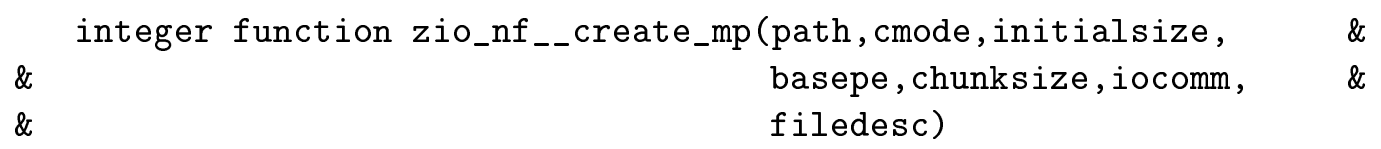

\section{DESCRIPTION:}

Creates a netCDF file using I/O staging communicator object iocomm. Returns the created file descriptor in filedesc. Specific to the parallel netCDF library being developed at NERSC.

INPUT PARAMETERS:

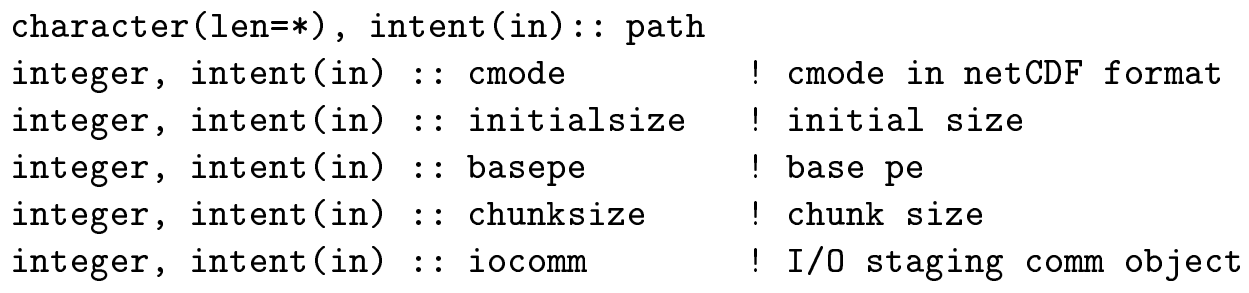

OUTPUT PARAMETERS:

integer, intent(out): : filedesc

\subsection{4 zio_nf__open}

INTERFACE:

$$
\text { \& integer function zio_nf_-open(path,omode, chunksize,iocomm, \& }
$$

\section{DESCRIPTION:}

Opens a netCDF file using I/O staging communicator object iocomm. Returns the created file descriptor in filedesc. Specific to the parallel netCDF library being developed at NERSC. 
character (len $=*$ ), intent(in) : : path

integer, intent(in) $:$ : omode

integer, intent(in) : : chunksize

integer, intent(in) : : iocomm

OUTPUT PARAMETERS:

integer, intent(out): : filedesc
! omode in netCDF format

! chunk size

! I/O staging comm object

\subsection{5 zio_nf__open_mp}

INTERFACE:

$$
\&
$$$$
\text { integer function zio_nf__open_mp(path,omode, basepe, chunksize, \& }
$$
iocomm,filedesc)

\section{DESCRIPTION:}

Opens a netCDF file using I/O staging communicator object iocomm. Returns the created file descriptor in filedesc. Specific to the parallel netCDF library being developed at NERSC.

INPUT PARAMETERS:

character (len=*), intent(in):: path integer, intent(in) $:$ : omode ! omode in netCDF format integer, intent(in) : : basepe ! base pe integer, intent(in) :: chunksize ! chunk size integer, intent(in) : : iocomm ! I/0 staging comm object

OUTPUT PARAMETERS:

integer, intent(out): : filedesc

\subsection{6 zio_nf_abort}

\section{INTERFACE:}

integer function zio_nf_abort(filedesc)

\section{DESCRIPTION:}

Backs out of recent definitions.

INPUT PARAMETERS:

integer, intent(in):: filedesc 


\subsection{7 zio_nf_close}

INTERFACE:

integer function zio_nf_close(filedesc)

DESCRIPTION:

Closes a netCDF file.

INPUT PARAMETERS:

integer, intent(in): : filedesc

\subsection{8 zio_nf_copy_att}

INTERFACE:

integer function zio_nf_copy_att(filedesc_in,varid_in, name, \& $\&$

filedesc_out,varid_out)

\section{DESCRIPTION:}

Copys attribute

INPUT PARAMETERS:

integer, intent(in):: filedesc_in

integer, intent(in): : varid_in

character(len=*), intent(in) : : name

integer, intent(in): : filedesc_out

integer, intent(in): : varid_out

\subsection{9 zio_nf_create}

INTERFACE:

integer function zio_nf_create(path, cmode, iocomm,filedesc)

DESCRIPTION:

Creates a netCDF file using I/O staging communicator object iocomm. Returns the created file descriptor in filedesc.

INPUT PARAMETERS: 
character (len $=*)$, intent(in) : : path

integer, intent(in) $:$ : cmode

integer, intent(in) : : iocomm

OUTPUT PARAMETERS:

integer, intent(out): : filedesc
! cmode in netCDF format

! I/O comm obj

\subsubsection{0 zio_nf_def_dim}

\section{INTERFACE:}

integer function zio_nf_def_dim(filedesc,name, length,dimid)

DESCRIPTION:

Defines a netCDF dimension.

INPUT PARAMETERS:

integer, intent(in):: filedesc

integer, intent(in): : length

character(len=*), intent(in):: name

OUTPUT PARAMETERS:

integer, intent(out): : dimid

\subsubsection{1 zio_nf_def_var}

INTERFACE:

integer function zio_nf_def_var(filedesc,name,xtype,nvdims, \& \& vdims, varid)

\section{DESCRIPTION:}

Defines a netCDF variable.

INPUT PARAMETERS:

integer, intent(in): : filedesc

integer, intent(in):: xtype

integer, intent(in): : nvdims

integer, intent(in):: vdims $(*)$

character(len $=*$ ), intent(in): : name

OUTPUT PARAMETERS:

integer, intent(out): :varid 


\subsubsection{2 zio_nf_del_att}

INTERFACE:

integer function zio_nf_del_att (filedesc,varid, name)

DESCRIPTION:

Deletes an attribute for a netCDF variable.

INPUT PARAMETERS:

integer, intent(in): : filedesc

integer, intent(in): : varid

character (len $=*$ ), intent(in): : name

\subsubsection{3 zio_nf_enddef}

INTERFACE:

integer function zio_nf_enddef(filedesc)

DESCRIPTION:

Ends the define mode.

INPUT PARAMETERS:

integer, intent(in): : filedesc

\subsubsection{4 zio_nf_get_att_double}

INTERFACE:

$$
\text { \& integer function zio_nf_get_att_double(filedesc, varid,name, } \text { dvals })_{\text {\& }}
$$

\section{DESCRIPTION:}

Returns the real*8 attribute values for a netCDF variable.

INPUT PARAMETERS:

integer, intent(in): : filedesc

integer, intent(in): : varid

character (len $=*$ ), intent(in): : name

OUTPUT PARAMETERS:

real(zio_r8), intent(out) :: $\operatorname{dvals}(*)$ 


\subsubsection{5 zio_nf_get_att_int}

INTERFACE:

integer function zio_nf_get_att_int(filedesc, varid, name,ivals)

DESCRIPTION:

Returns the integer*4 attribute values for a netCDF variable.

INPUT PARAMETERS:

integer, intent(in): : filedesc

integer, intent(in): : varid

character (len $=*$ ), intent(in): : name

OUTPUT PARAMETERS:

integer(zio_i4), intent(out) : : ivals(*)

\subsubsection{6 zio_nf_get_att_real}

INTERFACE:

integer function zio_nf_get_att_real(filedesc, varid, name,rvals)

DESCRIPTION:

Returns the real*4 attribute values for a netCDF variable.

INPUT PARAMETERS:

integer, intent(in): : filedesc

integer, intent(in): : varid

character(len $=*$ ), intent(in): : name

OUTPUT PARAMETERS:

real(zio_r4), intent(out) : : rvals $(*)$

\subsubsection{7 zio_nf_get_att_text}

INTERFACE:

integer function zio_nf_get_att_text(filedesc,varid, name,text) 
DESCRIPTION:

Returns the attribute text from the given variable ID and attribute name.

INPUT PARAMETERS:

integer, intent(in): : filedesc

integer, intent(in): : varid

character(len $=*$ ), intent(in): : name

OUTPUT PARAMETERS:

character (len=*), intent (out) : : text

\subsubsection{8 zio_nf_get_var_double}

INTERFACE:

integer function zio_nf_get_var_double(filedesc,varid,dvals, \&

$\&$ distarray)

DESCRIPTION:

Gets the given real*8 variable from an input file.

INPUT PARAMETERS:

integer, intent(in) : : filedesc

integer, intent(in) : : varid

integer, optional, intent(in) : : distarray

OUTPUT PARAMETERS:

real(zio_r8), intent(out) : $\operatorname{dvals}(*)$

\subsubsection{9 zio_nf_get_var_int}

INTERFACE:

\section{$\&$}

integer function zio_nf_get_var_int(filedesc,varid,ivals, distarray)

\section{DESCRIPTION:}

Gets the given integer*4 variable from an input file.

INPUT PARAMETERS: 
integer, intent(in) : : filedesc

integer, intent(in) : : varid

integer, optional, intent(in) : : distarray

OUTPUT PARAMETERS:

integer(zio_i4), intent(out) : : ivals(*)

\subsubsection{0 zio_nf_get_var_real}

INTERFACE:

\& integer function zio_nf_get_var_real(filedesc,varid,rvals, $\quad$ distarray)

\section{DESCRIPTION:}

Gets the given real*4 variable from an input file.

INPUT PARAMETERS:

integer, intent(in) : : filedesc

integer, intent(in) : : varid

integer, optional, intent(in) : : distarray

OUTPUT PARAMETERS:

real(zio_r4), intent(out) : : rvals $(*)$

\subsubsection{1 zio_nf_get_var_text}

INTERFACE:

integer function zio_nf_get_var_text(filedesc,varid,text)

DESCRIPTION:

Gets the given text variable from an input file. Note that text is not distributed.

INPUT PARAMETERS:

integer, intent(in) : : filedesc

integer, intent(in) : : varid

OUTPUT PARAMETERS:

character(len=*), intent(out) : : text 


\subsubsection{2 zio_nf_get_var1_double}

INTERFACE:

$$
\text { \& integer function zio_nf_get_var1_double(filedesc,varid,indx, \& }
$$

\section{DESCRIPTION:}

Gets the given real $* 8$ variable from the specified location of an input file.

INPUT PARAMETERS:

integer, intent(in) : : filedesc

integer, intent(in) : : indx $(*)$

integer, intent(in) : : varid

OUTPUT PARAMETERS:

real(zio_r8), intent(out) : : dval

\subsubsection{3 zio_nf_get_var1_int}

INTERFACE:

integer function zio_nf_get_var1_int(filedesc, varid,indx, ival)

DESCRIPTION:

Gets the given integer*4 variable from the specified location of an input file.

INPUT PARAMETERS:

integer, intent(in) : : filedesc

integer, intent(in) : : indx $(*)$

integer, intent(in) : : varid

OUTPUT PARAMETERS:

integer(zio_i4), intent(out) : : ival

\subsubsection{4 zio_nf_get_var1_real}

INTERFACE:

integer function zio_nf_get_var1_real(filedesc,varid,indx,rval) 
DESCRIPTION:

Gets the given real*4 variable from the specified location in of an input file.

INPUT PARAMETERS:

integer, intent(in) : : filedesc

integer, intent(in) : : indx $(*)$

integer, intent(in) : : varid

OUTPUT PARAMETERS:

real(zio_r4), intent(out) : : rval

\subsubsection{5 zio_nf_get_var1_text}

INTERFACE:

integer function zio_nf_get_var1_text(filedesc,varid,indx,chval)

\section{DESCRIPTION:}

Gets the given text variable from the specified location of an input file.

INPUT PARAMETERS:

integer, intent(in) : : filedesc

integer, intent(in) : : indx $(*)$

integer, intent(in) : : varid

OUTPUT PARAMETERS:

character, intent(out) : : chval

\subsubsection{6 zio_nf_get_vara_double}

INTERFACE:

$$
\&
$$$$
\text { integer function zio_nf_get_vara_double(filedesc,varid,istart, \& }
$$

icount, dvals, distarray)

\section{DESCRIPTION:}

Gets a range of the given real*8 variable from an input file.

INPUT PARAMETERS: 


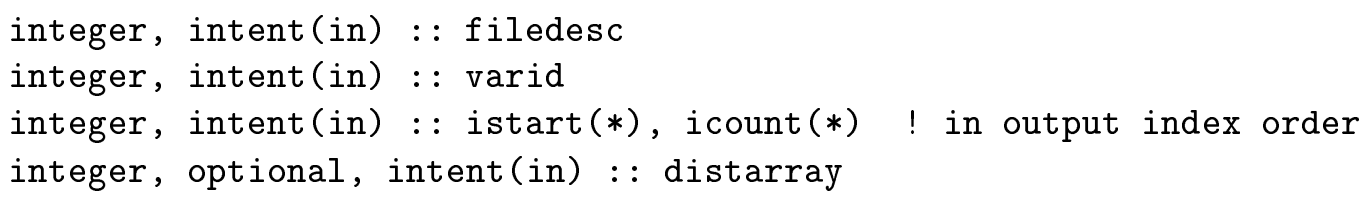

OUTPUT PARAMETERS:

real(zio_r8), intent(out) : : $\operatorname{dvals}(*)$

REMARKS: istart and icount used to refer only to the array section in the netCDF data space in disk, but not array's memory space. However, currently we let istart and icount refer to an array section in both the netCDF data and memory space. That is, an array section in netCDF data space is written from or read into the corresponding section of the global array.

\subsubsection{7 zio_nf_get_vara_int}

INTERFACE:

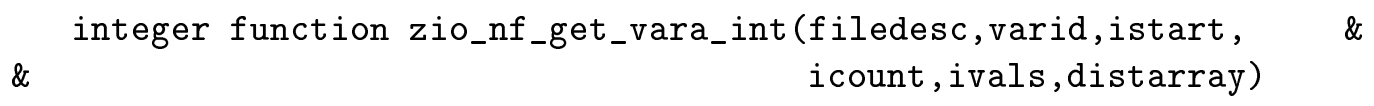

\section{DESCRIPTION:}

Gets a range of the given integer*4 variable from an input file.

INPUT PARAMETERS:

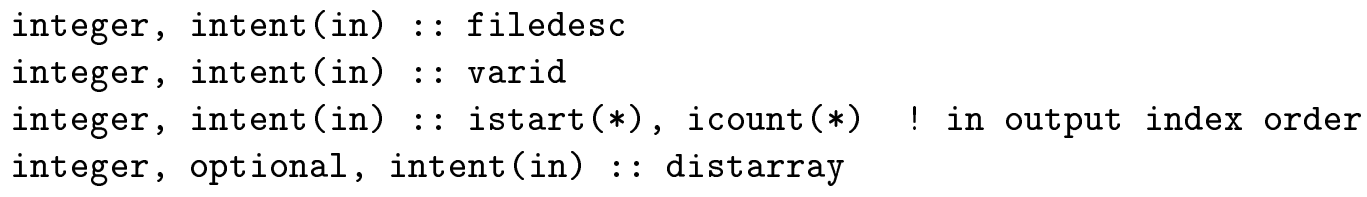

OUTPUT PARAMETERS:

integer(zio_i4), intent(out) : : ivals (*)

REMARKS: istart and icount used to refer only to the array section in the netCDF data space in disk, but not array's memory space. However, currently we let istart and icount refer to an array section in both the netCDF data and memory space. That is, an array section in netCDF data space is written from or read into the corresponding section of the global array.

\subsubsection{8 zio_nf_get_vara_real}

INTERFACE:

integer function zio_nf_get_vara_real(filedesc,varid,istart, \& 


\section{DESCRIPTION:}

Gets a range of the given real $* 4$ variable from an input file.

INPUT PARAMETERS:

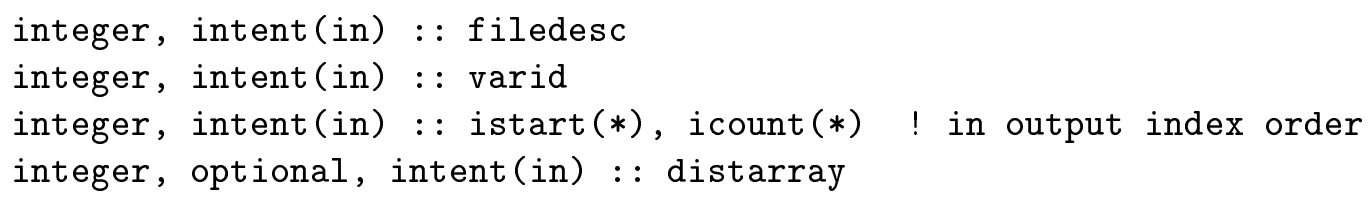

INPUT PARAMETERS:

real(zio_r4), intent(out) : : rvals $(*)$

REMARKS: istart and icount used to refer only to the array section in the netCDF data space in disk, but not array's memory space. However, currently we let istart and icount refer to an array section in both the netCDF data and memory space. That is, an array section in netCDF data space is written from or read into the corresponding section of the global array.

\subsubsection{9 zio_nf_get_vara_text}

INTERFACE:

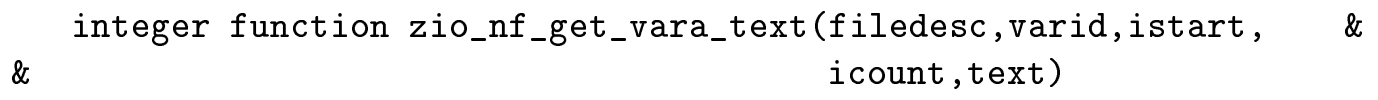

DESCRIPTION:

Gets a range of the given text variable from an input file. Note that text is not distributed.

INPUT PARAMETERS:

integer, intent(in) : : filedesc

integer, intent(in) : : varid

integer, intent(in) : : istart $(*)$, icount $(*)$

OUTPUT PARAMETERS:

character (len=*), intent(out) : : text

\subsubsection{0 zio_nf_inq}

INTERFACE: 


\section{DESCRIPTION:}

Gets number of dimensions, number of variables, number of attributes and unlimited dimension ID.

INPUT PARAMETERS:

integer, intent(in): : filedesc

OUTPUT PARAMETERS:

integer, intent(out): : ndims

integer, intent(out): : nvars

integer, intent(out): : ngatts

integer, intent(out): : unlimdimid

\subsubsection{1 zio_nf_inq_att}

INTERFACE:

integer function zio_nf_inq_att(filedesc,varid, name, xtype,

\section{DESCRIPTION:}

Gets information about an attribute.

INPUT PARAMETERS:

integer, intent(in):: filedesc

integer, intent(in): : varid

character(len $=*$ ), intent(in): : name

OUTPUT PARAMETERS:

integer, intent(out): : xtype

integer, intent(out): : length

\subsubsection{2 zio_nf_inq_attid}

INTERFACE:

integer function zio_nf_inq_attid(filedesc, varid, name, attnum)

DESCRIPTION:

Gets the attribute number. 
integer, intent(in): : filedesc

integer, intent(in): : varid

character (len $=*$ ), intent(in): : name

OUTPUT PARAMETERS:

integer, intent(out): : attnum

\subsubsection{3 zio_nf_inq_attlen}

INTERFACE:

integer function zio_nf_inq_attlen(filedesc, varid, name, length)

DESCRIPTION:

Gets the number of values (or characters) stored for an attribute.

INPUT PARAMETERS:

integer, intent(in): : filedesc

integer, intent(in): : varid

character (len $=*$ ), intent(in): : name

OUTPUT PARAMETERS:

integer, intent(out): : length

\subsubsection{4 zio_nf_inq_attname}

INTERFACE:

integer function zio_nf_inq_attname(filedesc, varid, attnum, name)

\section{DESCRIPTION:}

Gets attribute name.

INPUT PARAMETERS:

integer, intent(in): : filedesc

integer, intent(in): : varid

integer, intent(in): : attnum

OUTPUT PARAMETERS:

character (len=*), intent (out): : name 


\subsubsection{5 zio_nf_inq_atttype}

INTERFACE:

integer function zio_nf_inq_atttype(filedesc,varid, name, xtype)

DESCRIPTION:

Gets the attribute type.

INPUT PARAMETERS:

integer, intent(in): : filedesc

integer, intent(in): : varid

character (len $=*$ ), intent(in): : name

OUTPUT PARAMETERS:

integer, intent(out): : xtype

\subsubsection{6 zio_nf_inq_base_pe}

INTERFACE:

integer function zio_nf_inq_base_pe(filedesc,basepe)

DESCRIPTION:

Gets the base PE. Specific to the parallel netCDF library being developed at NERSC.

INPUT PARAMETERS:

integer, intent(in):: filedesc

OUTPUT PARAMETERS:

integer, intent(out):: basepe

\subsubsection{7 zio_nf_inq_dim}

INTERFACE:

integer function zio_nf_inq_dim(filedesc,dimid, name, length)

DESCRIPTION:

Gets dimension name and length for a given dimension ID. 
integer, intent(in): : filedesc

integer, intent(in): : dimid

OUTPUT PARAMETERS:

integer, intent(out): : length

character (len $=*$ ), intent (out): : name

\subsubsection{8 zio_nf_inq_dimid}

INTERFACE:

integer function zio_nf_inq_dimid(filedesc, name,dimid)

\section{DESCRIPTION:}

Gets the dimension ID for a given dimension name.

INPUT PARAMETERS:

integer, intent(in): : filedesc

character(len $=*$ ), intent(in): : name

OUTPUT PARAMETERS:

integer, intent(out): : dimid

\subsubsection{9 zio_nf_inq_dimlen}

INTERFACE:

integer function zio_nf_inq_dimlen(filedesc,dimid,length)

\section{DESCRIPTION:}

Gets the dimension length for a given dimension.

INPUT PARAMETERS:

integer, intent(in): : filedesc

integer, intent(in): : dimid

OUTPUT PARAMETERS:

integer, intent(out): : length 


\subsubsection{0 zio_nf_inq_dimname}

INTERFACE:

integer function zio_nf_inq_dimname(filedesc,dimid,name)

\section{DESCRIPTION:}

Gets dimension name for a given dimension ID.

INPUT PARAMETERS:

integer, intent(in): : filedesc

integer, intent(in): : dimid

OUTPUT PARAMETERS:

character (len $=*$ ), intent (out): : name

\subsubsection{1 zio_nf_inq_libvers}

INTERFACE:

function zio_nf_inq_libvers()

DESCRIPTION:

Get netCDF library version.

OUTPUT PARAMETERS:

character (len=80) : : zio_nf_inq_libvers

\subsubsection{2 zio_nf_inq_natts}

INTERFACE:

integer function zio_nf_inq_natts(filedesc,ngatts)

DESCRIPTION:

Returns the number of global attributes.

INPUT PARAMETERS:

integer, intent(in): : filedesc

OUTPUT PARAMETERS:

integer, intent(out): : ngatts 


\subsubsection{3 zio_nf_inq_ndims}

INTERFACE:

integer function zio_nf_inq_ndims(filedesc,ndims)

DESCRIPTION:

Returns the number of dimensions.

INPUT PARAMETERS:

integer, intent(in): : filedesc

OUTPUT PARAMETERS:

integer, intent(out): : ndims

\subsubsection{4 zio_nf_inq_nvars}

INTERFACE:

integer function zio_nf_inq_nvars(filedesc,nvars)

DESCRIPTION:

Returns the number of variables.

INPUT PARAMETERS:

integer, intent(in): : filedesc

OUTPUT PARAMETERS:

integer, intent(out): : nvars

\subsubsection{5 zio_nf_inq_unlimdim}

INTERFACE:

integer function zio_nf_inq_unlimdim(filedesc,unlimdimid)

DESCRIPTION:

Returns the unlimited dimension ID.

INPUT PARAMETERS:

integer, intent(in): : filedesc

OUTPUT PARAMETERS:

integer, intent(out): : unlimdimid 


\subsubsection{6 zio_nf_inq_var}

INTERFACE:

$$
\begin{array}{r}
\text { integer function zio_nf_inq_var(filedesc,varid, name, } x \text { type, } \\
\text { ndims, dimids, natts) }
\end{array}
$$

\section{DESCRIPTION:}

Returns the variable name, type, number of dimensions, dimension ID's, and number of attributes.

INPUT PARAMETERS:

integer, intent(in): : filedesc

integer, intent(in): : varid

OUTPUT PARAMETERS:

integer, intent(out): : xtype

integer, intent(out): : ndims

integer, intent(out): : dimids $(*)$

integer, intent(out) $:$ : natts

character (len=*), intent (out): : name

\subsubsection{7 zio_nf_inq_vardimid}

INTERFACE:

integer function zio_nf_inq_vardimid(filedesc,varid,dimids)

DESCRIPTION:

Returns the dimension ID's from a variable.

INPUT PARAMETERS:

integer, intent(in): : filedesc

integer, intent(in): : varid

OUTPUT PARAMETERS:

integer, intent(out):: $\operatorname{dimids}(*)$ 


\subsubsection{8 zio_nf_inq_varid}

INTERFACE:

integer function zio_nf_inq_varid(filedesc,name,varid)

DESCRIPTION:

Returns the variable ID for a given variable name.

INPUT PARAMETERS:

integer, intent(in): : filedesc

character(len $=*$ ), intent(in): : name

OUTPUT PARAMETERS:

integer, intent(out): : varid

\subsubsection{9 zio_nf_inq_varname}

INTERFACE:

integer function zio_nf_inq_varname(filedesc,varid,name)

\section{DESCRIPTION:}

Returns the variable name from the variable ID.

INPUT PARAMETERS:

integer, intent(in): : filedesc

integer, intent(in): : varid

OUTPUT PARAMETERS:

character (len=*), intent (out): : name

\subsubsection{0 zio_nf_inq_varnatts}

INTERFACE:

integer function zio_nf_inq_varnatts(filedesc,varid, natts)

DESCRIPTION:

Returns the number of attributes from the variable ID. 
integer, intent(in): : filedesc

integer, intent(in):: varid

OUTPUT PARAMETERS:

integer, intent(out): : natts

\subsubsection{1 zio_nf_inq_varndims}

INTERFACE:

integer function zio_nf_inq_varndims(filedesc,varid,ndims)

DESCRIPTION:

Returns the number of variable dimensions from the variable ID.

INPUT PARAMETERS:

integer, intent(in):: filedesc

integer, intent(in): : varid

OUTPUT PARAMETERS:

integer, intent(out): : ndims

\subsubsection{2 zio_nf_inq_vartype}

INTERFACE:

integer function zio_nf_inq_vartype(filedesc,varid, xtype)

\section{DESCRIPTION:}

Returns the variable type from the variable ID.

INPUT PARAMETERS:

integer, intent(in):: filedesc

integer, intent(in):: varid

OUTPUT PARAMETERS:

integer, intent(out): : xtype 


\subsubsection{3 zio_nf_open}

INTERFACE:

integer function zio_nf_open(path,omode, iocomm,filedesc)

\section{DESCRIPTION:}

Opens a netCDF file using I/O staging communicator object iocomm. Returns the created file descriptor in filedesc.

INPUT PARAMETERS:

character (len=*), intent(in):: path

integer, intent(in) : omode ! omode in netCDF format

integer, intent(in) : : iocomm I/O staging comm object

OUTPUT PARAMETERS:

integer, intent(out): : filedesc

\subsubsection{4 zio_nf_put_att_double}

INTERFACE:

integer function zio_nf_put_att_double(filedesc,varid, name, \& $\&$ xtype, length, dvals)

DESCRIPTION:

Puts the given real $* 8$ attribute values to variable ID.

INPUT PARAMETERS:

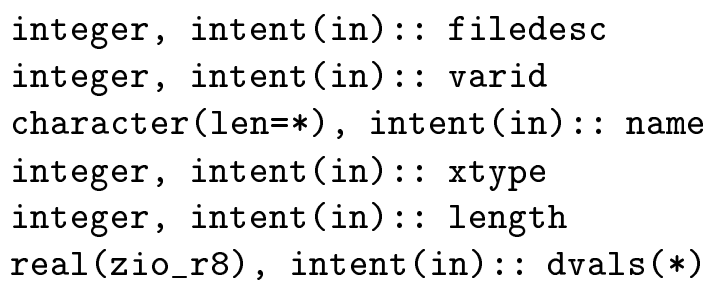

\subsubsection{5 zio_nf_put_att_int}

INTERFACE: 


\section{DESCRIPTION:}

Puts the given integer $* 4$ attribute value to variable ID.

INPUT PARAMETERS:

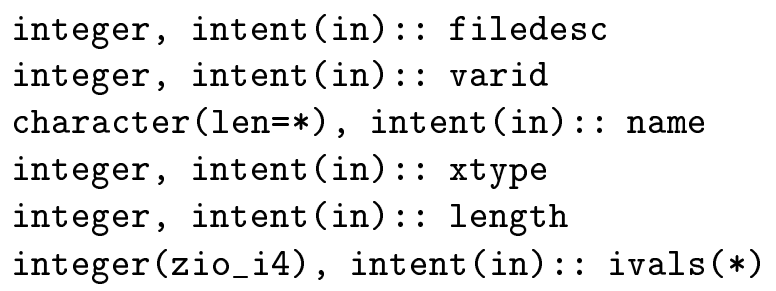

\subsubsection{6 zio_nf_put_att_real}

INTERFACE:

$$
\&
$$

integer function zio_nf_put_att_real(filedesc,varid,name,

\section{DESCRIPTION:}

Puts the given real $* 4$ attribute value to variable ID.

INPUT PARAMETERS:

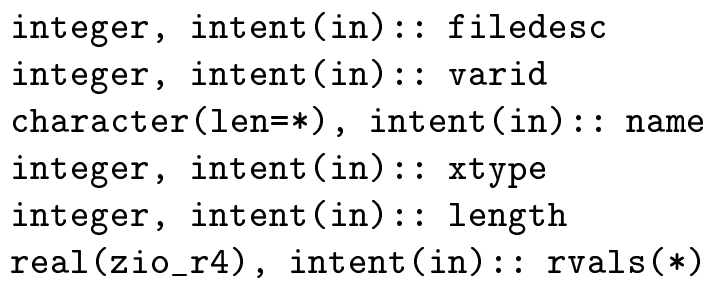

\subsubsection{7 zio_nf_put_att_text}

INTERFACE:

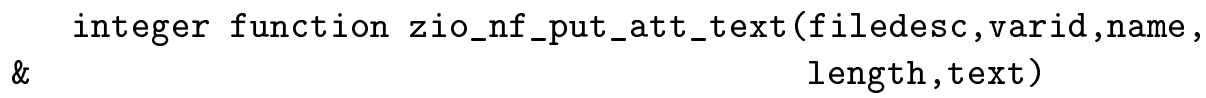




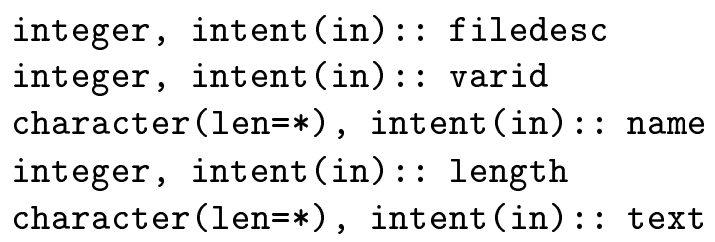

\subsubsection{8 zio_nf_put_var_double}

\section{INTERFACE:}

$$
\text { \& integer function zio_nf_put_var_double(filedesc,varid,dvals, } \quad \text { distarray) }
$$

\section{DESCRIPTION:}

Puts the given $r e a l * 8$ variable to an output file.

INPUT PARAMETERS:

integer, intent(in) :: filedesc

integer, intent(in) :: varid

real(zio_r8), intent(in) : : dvals $(*)$

integer, optional, intent(in) : : distarray

\subsubsection{9 zio_nf_put_var_int}

INTERFACE:

$$
\text { \& integer function zio_nf_put_var_int(filedesc, varid,ivals, }
$$

\section{DESCRIPTION:}

Puts the given integer $* 4$ variable to an output file.

INPUT PARAMETERS:

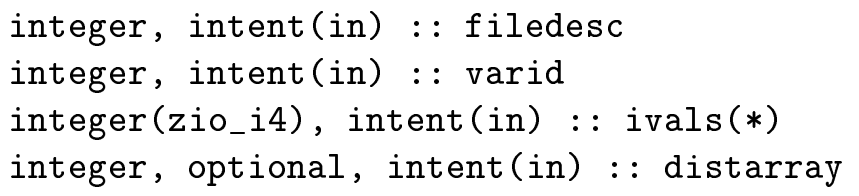




\subsubsection{0 zio_nf_put_var_real}

INTERFACE:

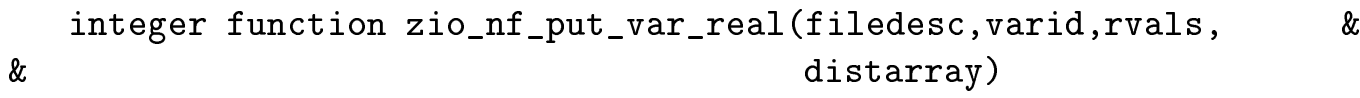

\section{DESCRIPTION:}

Puts the given real*4 variable to an output file.

INPUT PARAMETERS:

integer, intent(in) : : filedesc

integer, intent(in) : : varid

real(zio_r4), intent(in) : : rvals $(*)$

\subsubsection{1 zio_nf_put_var_text}

INTERFACE:

integer function zio_nf_put_var_text(filedesc,varid,text)

\section{DESCRIPTION:}

Puts the given text variable to an output file. Note that text is not distributed.

INPUT PARAMETERS:

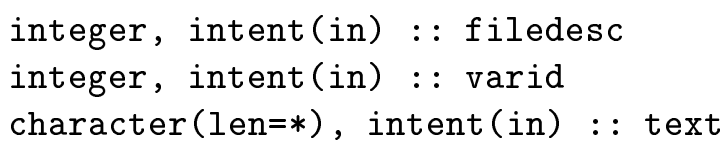

\subsubsection{2 zio_nf_put_var1_double}

INTERFACE:

integer function zio_nf_put_var1_double(filedesc,varid,indx, \& $\&$ dval)

\section{DESCRIPTION:}

Puts the given real $* 8$ variable at the specified location in an output file.

INPUT PARAMETERS:

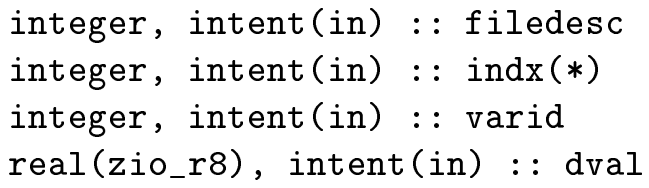




\subsubsection{3 zio_nf_put_var1_int}

INTERFACE:

integer function zio_nf_put_var1_int(filedesc,varid,indx,ival)

DESCRIPTION:

Puts the given integer $* 4$ variable at the specified location in an output file.

INPUT PARAMETERS:

integer, intent(in) :: filedesc

integer, intent(in) : : indx $(*)$

integer, intent(in) : : varid

integer(zio_i4), intent(in) : : ival

\subsubsection{4 zio_nf_put_var1_real}

INTERFACE:

integer function zio_nf_put_var1_real(filedesc,varid, indx,rval)

DESCRIPTION:

Puts the given real*4 variable at the specified location in an output file.

INPUT PARAMETERS:

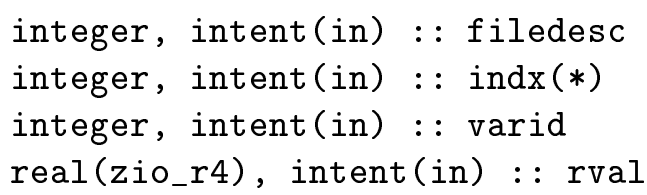

\subsubsection{5 zio_nf_put_var1_text}

INTERFACE:

integer function zio_nf_put_var1_text(filedesc,varid,indx,chval)

\section{DESCRIPTION:}

Puts the given character at the specified location in an output file.

INPUT PARAMETERS:

$$
\begin{aligned}
& \text { integer, intent(in) : filedesc } \\
& \text { integer, intent(in) : : indx(*) } \\
& \text { integer, intent(in) : : varid } \\
& \text { character, intent(in) : : chval }
\end{aligned}
$$




\subsubsection{6 zio_nf_put_vara_double}

INTERFACE:

$$
\begin{array}{r}
\text { integer function zio_nf_put_vara_double(filedesc, varid,istart, \& } \\
\text { icount,dvals, distarray) }
\end{array}
$$

DESCRIPTION:

Puts a range of the given real $* 8$ variable to an output file.

INPUT PARAMETERS:

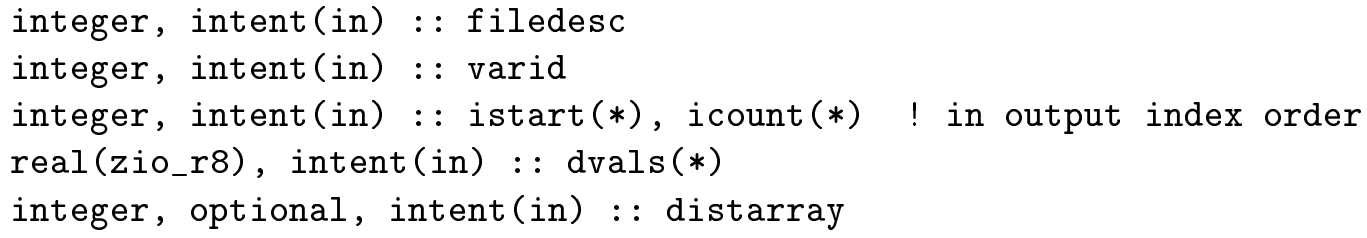

REMARKS: istart and icount used to refer only to the array section in the netCDF data space in disk, but not array's memory space. However, currently we let istart and icount refer to an array section in both the netCDF data and memory space. That is, an array section in netCDF data space is written from or read into the corresponding section of the global array.

\subsubsection{7 zio_nf_put_vara_int}

INTERFACE:

$$
\text { \& integer function zio_nf_put_vara_int(filedesc,varid,istart, } \quad \text { \& }
$$

DESCRIPTION:

Puts a range of the given integer*4 variable to an output file. Closes a netCDF file

INPUT PARAMETERS:

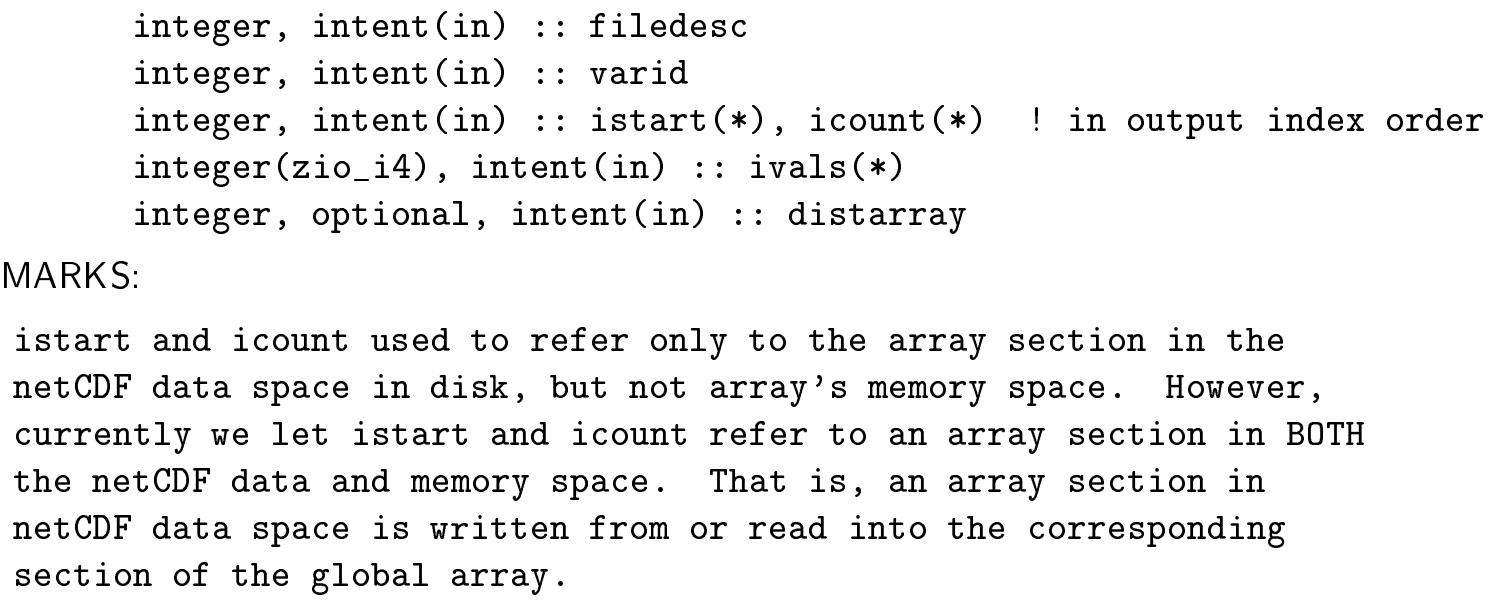

istart and icount used to refer only to the array section in the netCDF data space in disk, but not array's memory space. However, currently we let istart and icount refer to an array section in BOTH the netCDF data and memory space. That is, an array section in netCDF data space is written from or read into the corresponding section of the global array.

REMARKS: 


\subsubsection{8 zio_nf_put_vara_real}

INTERFACE:

$$
\begin{array}{r}
\text { integer function zio_nf_put_vara_real(filedesc, varid,istart, } \\
\text { icount,rvals, distarray) }
\end{array}
$$

\section{DESCRIPTION:}

Puts a range of the given real $* 4$ variable to an output file.

\section{INPUT PARAMETERS:}

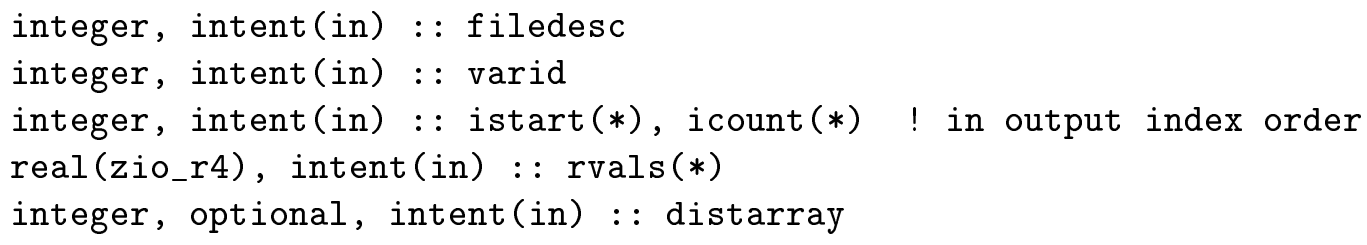

\section{REMARKS:}

istart and icount used to refer only to the array section in the netCDF data space in disk, but not array's memory space. However, currently we let istart and icount refer to an array section in BOTH the netCDF data and memory space. That is, an array section in netCDF data space is written from or read into the corresponding section of the global array.

\subsubsection{9 zio_nf_put_vara_text}

INTERFACE:

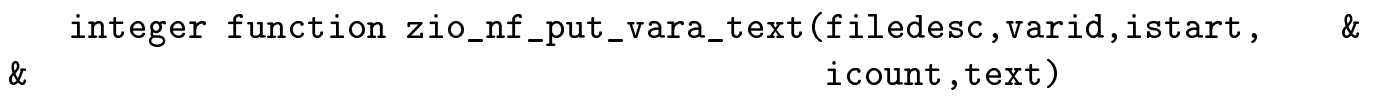

\section{DESCRIPTION:}

Puts a range of the given text variable to an output file. Note that text is not distributed.

INPUT PARAMETERS:

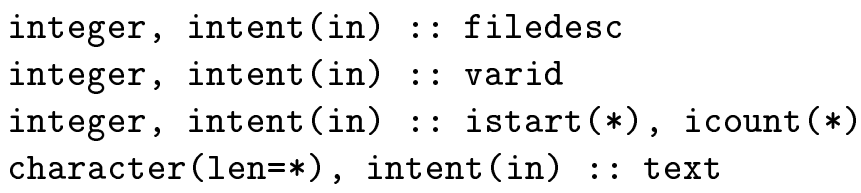




\subsubsection{0 zio_nf_redef}

INTERFACE:

integer function zio_nf_redef(filedesc)

DESCRIPTION:

Puts in the define mode.

INPUT PARAMETERS:

integer, intent(in): : filedesc

\subsubsection{1 zio_nf_rename_att}

INTERFACE:

integer function zio_nf_rename_att(filedesc,varid, name, newname)

DESCRIPTION:

Renames an attribute.

INPUT PARAMETERS:

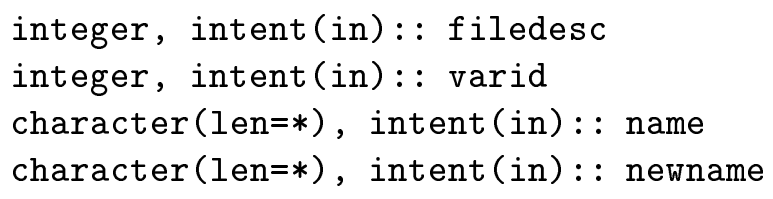

\subsubsection{2 zio_nf_rename_dim}

INTERFACE:

integer function zio_nf_rename_dim(filedesc,dimid,name)

DESCRIPTION:

Renames a dimension.

INPUT PARAMETERS:

integer, intent(in):: filedesc

character(len $=*$ ), intent(in): : name

OUTPUT PARAMETERS:

integer, intent(out): : dimid 


\subsubsection{3 zio_nf_rename_var}

INTERFACE:

integer function zio_nf_rename_var(filedesc,varid, newname)

DESCRIPTION:

Renames variable's name.

INPUT PARAMETERS:

integer, intent(in): : filedesc

character (len $=*$ ), intent (in): : newname

OUTPUT PARAMETERS:

integer, intent(out): : varid

\subsubsection{4 zio_nf_set_base_pe}

INTERFACE:

integer function zio_nf_set_base_pe(filedesc,basepe)

DESCRIPTION:

Resets the base PE. Specific to the parallel netCDF library being developed at NERSC.

INPUT PARAMETERS:

integer, intent(in): : filedesc

integer, intent(in):: basepe

\subsubsection{5 zio_nf_set_fill}

INTERFACE:

integer function zio_nf_set_fill(filedesc,fillmode,old_mode)

DESCRIPTION:

Sets the fill mode.

INPUT PARAMETERS:

integer, intent(in): : filedesc

integer, intent(in): : fillmode

OUTPUT PARAMETERS:

integer, intent(out): : old_mode 


\subsubsection{6 zio_nf_strerror}

INTERFACE:

function zio_nf_strerror(ncerr)

\section{DESCRIPTION:}

Returns an error message of len $=80$ for netCDF I/O error ncerr.

OUTPUT PARAMETERS:

character(len=80) : : zio_nf_strerror

INPUT PARAMETERS:

integer, intent(in) : : ncerr

\subsubsection{7 zio_nf_sync}

INTERFACE:

integer function zio_nf_sync(filedesc)

\section{DESCRIPTION:}

Synchronizes netCDF dataset to disk.

INPUT PARAMETERS:

integer, intent(in): : filedesc 